NASA

Technical

Paper

3031

1990

\section{Forbidden Tangential Orbit Transfers Between Intersecting Keplerian Orbits}

Rowland E. Burns

George C. Marshall Space Flight Center

Marshall Space Flight Center, Alabama 

TABLE OF CONTENTS

Page

INTRODUCTION

PROBLEM FORMULATION

FIRST SINGULAR CASE: HOHMANN TRANSFER

SECOND SINGULAR CASE: INITIAL ECCENTRICITY $=0$,

FINAL ECCENTRICITY $\neq 0$

THIRD SINGULAR CASE: INITIAL ECCENTRICITY $\neq 0$,

FINAL ECCENTRICITY $=0$

THE GENERAL CASE: INITIAL ECCENTRICITY $\neq 0$,

FINAL ECCENTRICITY $\neq 0$ 


\section{LIST OF SYMBOLS}

\section{Latin Symbols}

$\mathbf{a}_{\mathbf{i}}$

$$
e_{i}
$$

i

g

i

$p_{1} \cdot p_{2} \cdot p_{3}$

4

$Q_{i}$

$\mathbf{r}_{\mathrm{i}}$

r

$\mathrm{R}$

$\mathrm{x}$

Greek Symbols

$\alpha$

$\gamma$

$\varepsilon$

$\zeta$

$\vartheta$

$\theta$

$\xi$
Semi-major axis of orbit No. i

Eccentricity of orbit No. i

Generic function

Generic function

Dummy subscript of the range 1 to 3

Functions defined by equations (111), (112), and (113), respectively

Quantity defined by either equation (38) or by equation (86) depending upon the case under consideration

Quantity defined by equation (11)

Magnitude of radius vector or orbit No. i

Substitution made for algebraic convenience

Substitution made for algebraic convenience via equation (125)

Cartesian coordinate

Function defined by equation (119): the angular measure of the crossing points of the intersecting orbits.

Quantity defined by equation (95)

A positive real number which may be chosen to be arbitrarily small

Angular measure lunction defined by equation (138)

Rotation angle of the apogee of the target orbit

Generic angle

Angular measure function defined by equation (150) 
$\rho$

$\sigma$

$\tau$

$\phi_{\mathrm{i}}$

$\omega$

Special Symbols

ex

$+$

$-$

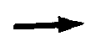

$\Longrightarrow$

$\bmod$
Rotation angle of the apogee of the transfer orbit

Angular measure function defined by equation (156)

Angular measure function defined by equation (157)

True anomaly of orbit No. i

Angular measure function defined by equation (40)

(Subscript) indicates an extremal value

(Superscript) indicates the value of a quantity just after the point in question

(Superscript) indicates the value of a quantity just before the point in question

Indicates a limiting value

Implication

Modulus 


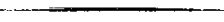


TECHNICAL PAPER

\title{
FORBIDDEN TANGENTIAL ORBIT TRANSFERS BETWEEN INTERSECTING KEPLERIAN ORBITS
}

\section{INTRODUCTION}

\begin{abstract}
Although the subject of impulsive transfers between Keplerian orbits has been treated in many papers (e.g. $[1,2]$ ), there has been no demonstration that certain transfers are not allowed. In this paper, it is shown that, for planar intersecting orbits, the tangential impulse transfer cannot always be utilized. The treatment is formulated as purely geometric in nature and no consideration is given to optimality.
\end{abstract}

For the two-impulse transfer between two coplanar elliptical orbits, there exists a triple infinity of elliptic orbits connecting the given orbits. In the case of realistic space transfer maneuvers, the optimum impulses are contained in a small useful angle between the tangential direction and the local horizontal direction as seen on the transfer orbit [3]. As a practical application, we may search for the optimum cotangential transfer as a sub-optimum solution.

The tangency conditions reduce the triple infinity of solutions to a single infinity of solutions so we are at liberty to parameterize these solutions by one convenient variable; the true anomaly of the initial orbit is chosen. It is then demonstrated that the descriptors of the transfer orbit can either be determined directly in terms of this parameter or eliminated from the discussion. Previous studies relied on a series of sequential calculations, and this obscured the fact that singularities can sometimes occur in the semi-major axis and eccentricities of the transfer orbit.

The arguments which exclude certain transfers for intersecting orbits are shown to be inapplicable for the case of non-intersecting orbits; singularities occur only for transfers between intersecting orbits.

I thank the (anonymous) reviewer who pointed out an interesting geometric argument based on the work of Professor Busemann [4] for Reference 5. The comments that were returned to me used a Busemann space construction to show that if the initial and final orbits do not intersect then the transfer solution is always available; in the case of intersecting orbits, forbidden transfer regions can occur. This entirely agrees with the results presented here.

The exact conditions for the occurrence of forbidden transfers are presented in this paper. Their existence provides a new result in a classical subject. 


\section{PROBLEM FORMULATION}

In the context of the (planar) Keplerian two body problem, define a cartesian coordinate system with the attracting primary at the origin. The initial orbit is oriented on this coordinate system in such a way that its perigee occurs along the $x$-axis. The initial orbit will be taken to have an eccentricity $e_{1}$ and a semimajor axis $a_{1}$. The final orbit has corresponding parameters $e_{3}$ and $a_{3}$ and is oriented in such a way that its semi-major axis is rotated clockwise through an angle $\vartheta$ from the negative $x$-axis. See Figure 1.

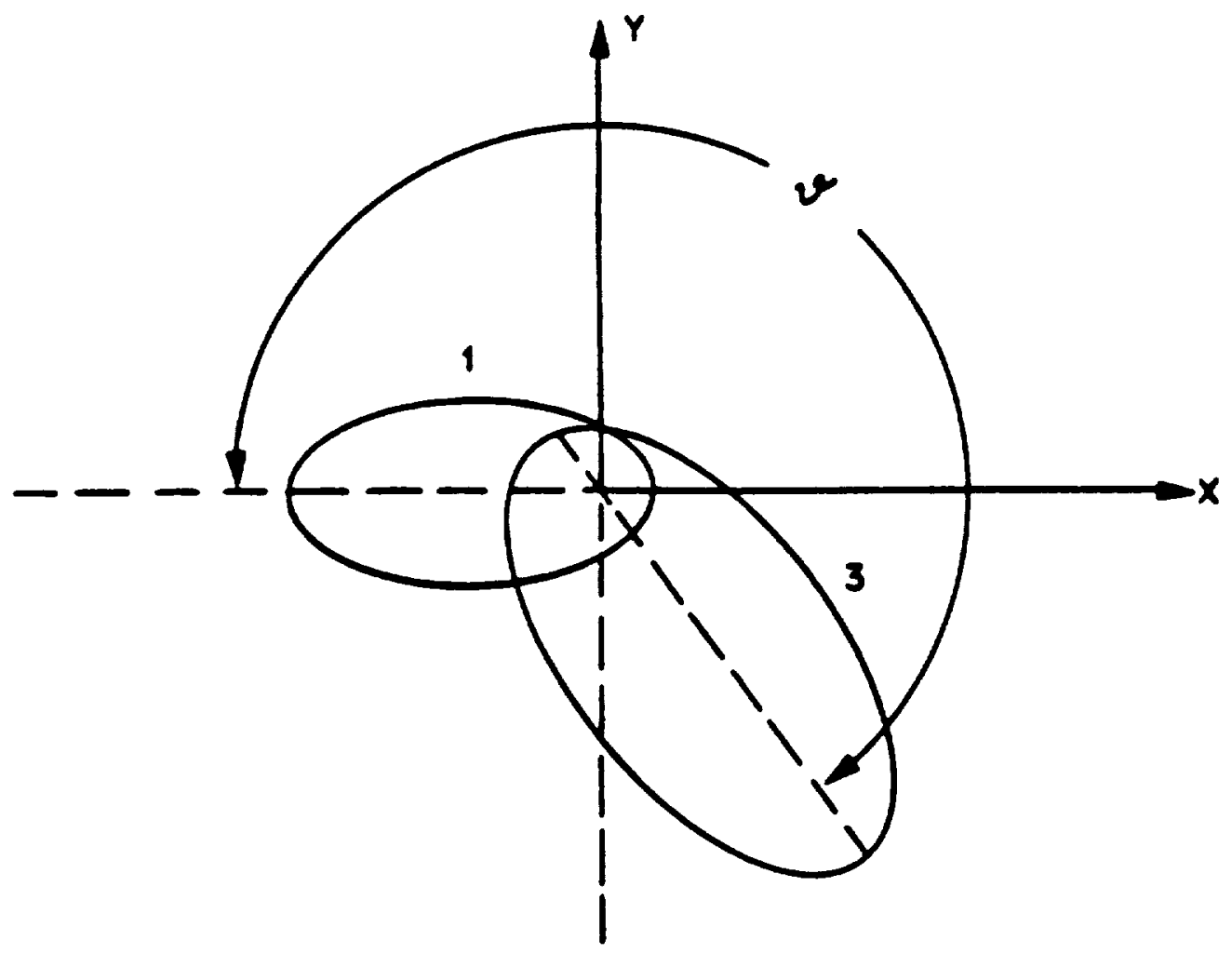

Figure 1

An orbit transfer from orbit No. 1 to orbit No. 3 can occur in many ways but only a subset of general transfers will be considered here, namely tangential transfers. The geometry of a transfer orbit can now be taken as equivalent to constructing a confocal conic section from some point, $\phi_{1}$, on the initial orbit to a point $\phi_{2}$ on the target orbit.

Four constraints exist, i.e., the radius of the transfer orbit must match at the initial and final points and the velocities must be colinear at the same points. The unknowns of the problem include the semi-major axis and eccentricity of the transfer orbit, the rotation (phase) angle of the transfer orbit, the angle $\phi_{1}$ (the angular leave point) and the angle $\phi_{2}$ (the angular arrival point). Since there are five unknowns and only four constraints, it is apparent that one free parameter exists and it is reasonable to parameterize with respect to the leave angle, $\phi_{1}$. Figure 2 shows a typical transfer orbit. 


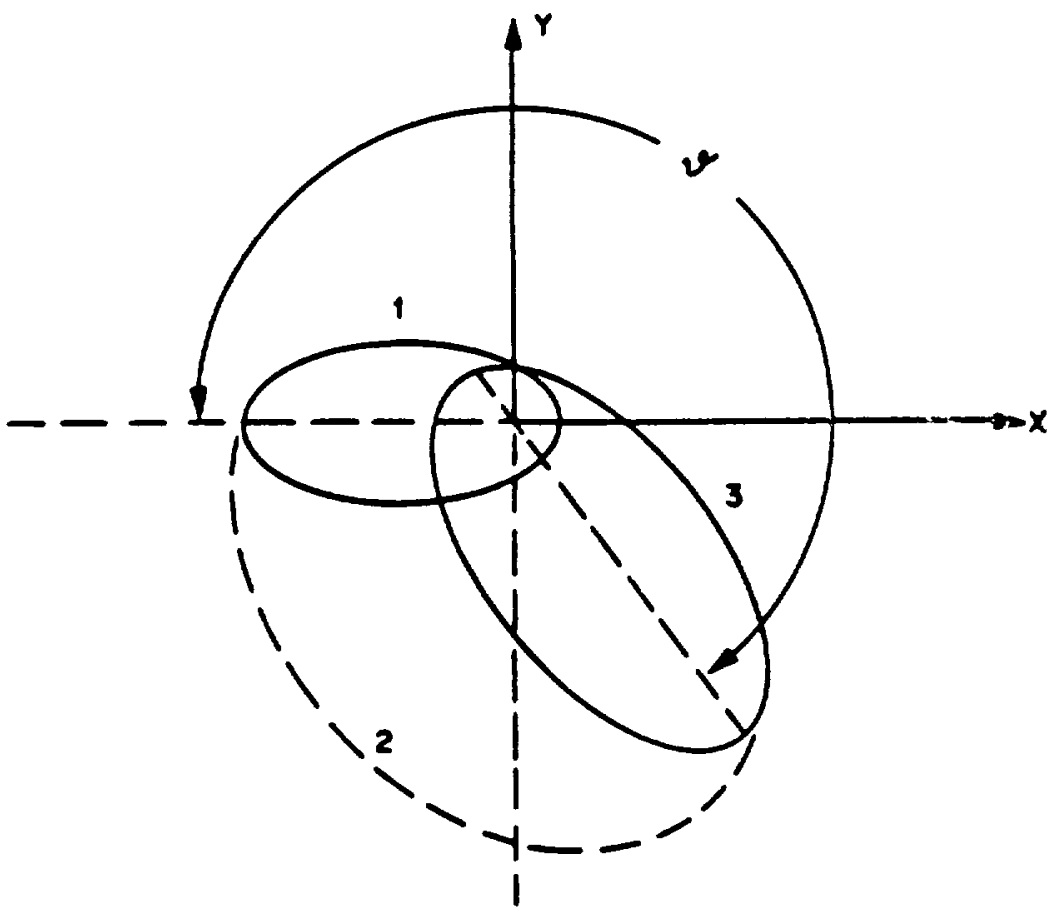

Figure 2.

To proceed with a quantitative formulation, the three orbits can be characterized by

$r_{1}=\frac{a_{1}\left(1-e_{1}^{2}\right)}{1+e_{1} \cos \phi}$

$r_{2}=\frac{a_{2}\left(1-e_{2}^{2}\right)}{1+e_{2} \cos (\phi+\rho)}$

$r_{3}=\frac{a_{3}\left(1-e_{3}^{2}\right)}{1+e_{3} \cos (\phi+\vartheta)}$

where $r_{1}, r_{2}, r_{3}$ are the radii of the three orbits, $a_{1}, a_{2}, a_{3}$ and $e_{1}, e_{2}, e_{3}$ are the semi-major axes and eccentricities of those orbits. The angle $\rho$ is the rotation angle of the apogee of the transfer orbit, $\vartheta$ is the rotation angle of the apogee of the final orbit, and $\phi$ is the true anomaly of the initial orbit.

From these

$$
\frac{\partial r_{1}}{\partial \phi}=\frac{r_{1}^{2} e_{1} \sin \phi}{a_{1}\left(1-e_{1}^{2}\right)}
$$




$$
\begin{aligned}
& \frac{\partial r_{2}}{\partial \phi}=\frac{r_{2}^{2} e_{2} \sin (\phi+\rho)}{a_{2}\left(1-e_{2}^{2}\right)} \\
& \frac{\partial r_{3}}{\partial \phi}=\frac{r_{3}^{2} e_{3} \sin (\phi+\vartheta)}{a_{3}\left(1-e_{3}{ }^{2}\right)}
\end{aligned}
$$

Equating the radii and requiring tangency at a generic point $\phi_{1}$ gives the first two constraint equations

$$
\begin{aligned}
& \frac{a_{1}\left(1-e_{1}^{2}\right)}{1+e_{1} \cos \phi_{1}}=\frac{a_{1}\left(1-e_{2}^{2}\right)}{1+e_{2} \cos \left(\phi_{1}+\rho\right)} \\
& \frac{r_{1}^{2} e_{1} \sin \phi_{1}}{a_{1}\left(1-e_{1}^{2}\right)}=\frac{r_{2}^{2} e_{2} \sin \left(\phi_{1}+\rho\right)}{a_{2}\left(1-e_{2}^{2}\right)}
\end{aligned}
$$

and the second two constaint equations come from a similar generic point $\phi_{2}$, i.e.,

$$
\begin{aligned}
& \frac{a_{2}\left(1-e_{2}^{2}\right)}{1+e_{2} \cos \left(\phi_{2}+\rho\right)}=\frac{a_{3}\left(1-e_{3}{ }^{2}\right)}{1+e_{3} \cos \left(\phi_{2}+\vartheta\right)} \\
& \frac{r_{2}^{2} e_{2} \sin \left(\phi_{2}+\rho\right)}{a_{2}\left(1-e_{2}{ }^{2}\right)}=\frac{r_{3}{ }^{2} e_{3} \sin \left(\phi_{2}+\vartheta\right)}{a_{3}\left(1-e_{3}{ }^{2}\right)}
\end{aligned}
$$

Since $r_{1}\left(\phi_{1}\right)=r_{2}\left(\phi_{1}\right)$ and $r_{2}\left(\phi_{2}\right)=r_{3}\left(\phi_{2}\right)$ and abbreviating

$$
\mathrm{Q}_{\mathrm{i}}=\frac{1}{\mathrm{a}_{\mathrm{i}}\left(1-\mathrm{e}_{\mathrm{i}}^{2}\right)}
$$

equations (7) through (10) can be written as

$$
\mathrm{Q}_{1}\left(1+\mathrm{e}_{1} \cos \phi_{1}\right)=\mathrm{Q}_{2}\left[1+\mathrm{e}_{2} \cos \left(\phi_{1}+\rho\right)\right]
$$




$$
\begin{aligned}
& \mathrm{Q}_{1} \mathrm{e}_{1} \sin \phi_{1}=\mathrm{Q}_{2} \mathrm{e}_{2} \sin \left(\phi_{1}+\rho\right) \\
& \mathrm{Q}_{2}\left[1+\mathrm{e}_{2} \cos \left(\phi_{2}+\rho\right)\right]=\mathrm{Q}_{3}\left[1+\mathrm{e}_{3} \cos \left(\phi_{2}+\vartheta\right)\right] \\
& \mathrm{Q}_{2} \mathrm{e}_{2} \sin \left(\phi_{2}+\rho\right)=\mathrm{Q}_{3} \mathrm{e}_{3} \sin \left(\phi_{2}+\vartheta\right)
\end{aligned}
$$

It should be noted that equations (12) through (15) constitute four equations in five unknowns, i.e., $\mathrm{Q}_{2}, \mathrm{e}_{2}, \rho, \phi_{2}$ and the parameter $\phi_{1}$.

The first solution to those equations which were obtained was the most general case, i.e., $e_{1} \neq 0$ and $e_{2} \neq 0$. Subsequently, it was found that the singular cases where either $e_{1}$ or $e_{3}$ (or $e_{1}$ and $e_{3}$ ) vanished should be treated separately. Since the derivation for singular cases is much easier than the general case, the order in which the solutions were obtained will be reversed, i.e., the presentation will start with $\mathrm{e}_{1}=\mathrm{e}_{3}=$ 0 (Hohmann transfer), then the two cases where either $\mathrm{e}_{1}=0$ or $\mathrm{e}_{3}=0$, and finally the general solution. It will be seen, later, that the general solution does not readily reduce to the special cases.

\section{First Singular Case: Hohmann Transfer}

Setting $\mathrm{e}_{1}=\mathrm{e}_{3}=0$, and, obviously, $\vartheta=0$, in equations (12) through (15) gives

$$
\begin{aligned}
& \mathrm{Q}_{1}=\mathrm{Q}_{2}\left[1+\mathrm{e}_{2} \cos \left(\phi_{1}+\rho\right)\right] \\
& \mathrm{O}=\mathrm{Q}_{2} \mathrm{e}_{2} \sin \left(\phi_{1}+\rho\right) \\
& \mathrm{Q}_{2}\left[1+\mathrm{e}_{2} \cos \left(\phi_{2}+\rho\right)\right]=\mathrm{Q}_{3} \\
& \mathrm{Q}_{2} \mathrm{e}_{2} \sin \left(\phi_{2}+\rho\right)=0 .
\end{aligned}
$$

Excluding $\mathrm{Q}_{2}=0, \mathrm{e}_{2}=0$, and $\mathrm{Q}_{1}=\mathrm{Q}_{3}$ immediately shows that, from (17) and (19)

$$
\begin{aligned}
& \cos \left(\phi_{1}+\rho\right)= \pm 1 \\
& \cos \left(\phi_{2}+\rho\right)= \pm 1
\end{aligned}
$$


which leaves the two equations

$$
\begin{aligned}
& \mathrm{Q}_{1}=\mathrm{Q}_{2}\left(1 \pm \mathrm{e}_{2}\right) \\
& \mathrm{Q}_{3}=\mathrm{Q}_{2}\left(1 \pm \mathrm{e}_{2}\right)
\end{aligned}
$$

Since $Q_{1} \neq Q_{3}$ the immediate conclusion is that there are two cases. Either

$$
\mathrm{Q}_{1}=\mathrm{Q}_{2}\left(1+\mathrm{e}_{2}\right)
$$

and

$$
\mathrm{Q}_{3}=\mathrm{Q}_{2}\left(1-\mathrm{e}_{2}\right)
$$

or

$$
\mathrm{Q}_{1}=\mathrm{Q}_{2}\left(1-\mathrm{e}_{2}\right)
$$

and

$$
\mathrm{Q}_{3}=\mathrm{Q}_{2}\left(1+\mathrm{e}_{2}\right)
$$

From the first case comes the solution

$$
\begin{aligned}
& \mathrm{e}_{2}=\frac{\mathrm{Q}_{1}-\mathrm{Q}_{3}}{\mathrm{Q}_{3}+\mathrm{Q}_{1}} \\
& \mathrm{Q}_{2}=\frac{\mathrm{Q}_{1}+\mathrm{Q}_{3}}{2}
\end{aligned}
$$

Since $e_{2}>0$, this solution applies whenever $Q_{1}>Q_{3}$. From the second case,

$$
\mathrm{e}_{2}=\frac{\mathrm{Q}_{3}-\mathrm{Q}_{1}}{\mathrm{Q}_{3}+\mathrm{Q}_{1}}
$$




$$
\mathrm{Q}_{2}=\frac{\mathrm{Q}_{1}+\mathrm{Q}_{3}}{2}
$$

which should be used when $\mathrm{Q}_{3}>\mathrm{Q}_{1}$.

$\phi_{1}$ is arbitrary, and for the transfer to be meaningful, $\rho= \pm \pi$. Then, $\phi_{2}= \pm \pi$.

\section{Second Singular Case: Initial Eccentricity $=0$, Final Eccentricity $\neq 0$}

Since $e_{1}=0$ the only thing which vitiates the spatial isotropy is the eccentricity of the target orbit. The rotation angle of the apogee of the target orbit can then be chosen to be equal to 0 (i.e., $\vartheta=0$ ) and equations (12) through (15) can be written as

$$
\begin{aligned}
& \mathrm{Q}_{1}=\mathrm{Q}_{2}\left[1+\mathrm{e}_{2} \cos \left(\phi_{1}+\rho\right)\right] \\
& 0=\mathrm{Q}_{2} \mathrm{e}_{2} \sin \left(\phi_{1}+\rho\right) \\
& \mathrm{Q}_{2}\left[1+\mathrm{e}_{2} \cos \left(\phi_{2}+\rho\right)\right]=\mathrm{Q}_{3}\left[1+\mathrm{e}_{3} \cos \phi_{2}\right] \\
& \mathrm{Q}_{2} \mathrm{e}_{2} \sin \left(\phi_{2}+\rho\right)=\mathrm{Q}_{3} \mathrm{e}_{3} \sin \phi_{2}
\end{aligned}
$$

From equation (25), either

$$
\rho=-\phi_{1}
$$

or

$$
\rho=\pi-\phi_{1}
$$

so two choices exist for $\rho$. Using a "parallel" derivation to reduce space produces the following. 


$$
\begin{aligned}
& \text { If } \rho=-\phi_{1} \text { then } \\
& Q_{1}=Q_{2}\left(1+e_{2}\right) \\
& Q_{2}\left[1+e_{2} \cos \left(\phi_{2}-\phi_{1}\right)\right]=Q_{3}\left[1+e_{3} \cos \phi_{2}\right] \\
& Q_{2} e_{2} \sin \left(\phi_{2}-\phi_{1}\right)=Q_{3} e_{3} \sin \phi_{2} \\
& \text { Dividing equation (32) by equation (31) yields } \\
& \frac{e_{2} \sin \left(\phi_{2}-\phi_{1}\right)}{I+e_{2} \cos \left(\phi_{2}-\phi_{1}\right)}=\frac{e_{3} \sin \phi_{2}}{1+e_{3} \cos \phi_{2}}
\end{aligned}
$$

Solving for $e_{2}$ yields

$$
\mathrm{e}_{2}=\frac{\mathrm{e}_{3} \sin \phi_{2}}{\sin \left(\phi_{2}-\phi_{1}\right)-\mathrm{e}_{3} \sin \phi_{1}}
$$

Then

$$
1+\mathrm{e}_{2}=\frac{\sin \left(\phi_{2}-\phi_{1}\right)+\mathrm{e}_{3}\left(\sin \phi_{2}-\sin \phi_{1}\right)}{\sin \left(\phi_{2}-\phi_{1}\right)-\mathrm{e}_{3} \sin \phi_{1}}
$$

From equation (30)

$Q_{2}=Q_{1}\left[\frac{\sin \left(\phi_{2}-\phi_{1}\right)-e_{3} \sin \phi_{1}}{\sin \left(\phi_{2}-\phi_{1}\right)+e_{3}\left(\sin \phi_{2}-\sin \phi_{1}\right)}\right]$

Inserting $e_{2}$ and $Q_{2}$ into (32) and clearing gives

$q \sin \left(\phi_{2}-\phi_{1}\right)-\sin \phi_{2}=-\sin \phi_{1}$
If $\rho=\pi-\phi_{1}$ then

$\mathrm{Q}_{1}=\mathrm{Q}_{2}\left(1-\mathrm{e}_{2}\right)$

$\mathrm{Q}_{2}\left[1-\mathrm{e}_{2} \cos \left(\phi_{2}-\phi_{1}\right)\right]=\mathrm{Q}_{3}\left[1+\mathrm{e}_{3} \cos \phi_{2}\right]$

$-\mathrm{Q}_{2} \mathrm{e}_{2} \sin \left(\phi_{2}-\phi_{1}\right)=\mathrm{Q}_{3} \mathrm{e}_{3} \sin \phi_{2}$

Dividing equation (32) by equation (31) yields

$\frac{-\mathrm{e}_{2} \sin \left(\phi_{2}-\phi_{1}\right)}{1-\mathrm{e}_{2} \cos \left(\phi_{2}-\phi_{1}\right)}=\frac{\mathrm{e}_{3} \sin \phi_{2}}{1+\mathrm{e}_{3} \cos \phi_{2}}$

Solving for $e_{2}$ yields

$\mathrm{e}_{2}=\frac{-\mathrm{e}_{3} \sin \phi_{2}}{\sin \left(\phi_{2}-\phi_{1}\right)-\mathrm{e}_{3} \sin \phi_{1}}$

Then

$1-\mathrm{e}_{2}=\frac{\sin \left(\phi_{2}-\phi_{1}\right)+\mathrm{e}_{3}\left(\sin \phi_{2}-\sin \phi_{1}\right)}{\sin \left(\phi_{2}-\phi_{1}\right)-\mathrm{e}_{3} \sin \phi_{1}}$

From equation (30)

$Q_{2}=Q_{1}\left[\frac{\sin \left(\phi_{2}-\phi_{1}\right)-e_{3} \sin \phi_{1}}{\sin \left(\phi_{2}-\phi_{1}\right)+\mathrm{e}_{3}\left(\sin \phi_{2}-\sin \phi_{1}\right)}\right]$

Inserting $\mathrm{e}_{2}$ and $\mathrm{Q}_{2}$ into (32) and clearing gives

$\mathrm{q} \sin \left(\phi_{2}-\phi_{1}\right)-\sin \phi_{2}=-\sin \phi_{1}$ 
where

$$
\mathrm{q}=\frac{\mathrm{Q}_{1}-\mathrm{Q}_{3}}{\mathrm{Q}_{3} \mathrm{e}_{3}}
$$

From (either) equation (37)

$$
\left(1-q \cos \phi_{1}\right) \sin \phi_{2}+\left(q \sin \phi_{1}\right) \cos \phi_{2}=\sin \phi_{1}
$$

Notice that equation (39) is satisfied by $\phi_{1}=\phi_{2}$, a solution specifically excluded by equation (36).

Setting

$$
\tan \omega=\frac{1-q \cos \phi_{1}}{q \sin \phi_{1}}
$$

so that

$$
\begin{aligned}
& \cos \omega=\frac{q \sin \phi_{1}}{\sqrt{q^{2}-2 q \cos \phi_{1}+1}} \\
& \sin \omega=\frac{1-q \cos \phi_{1}}{\sqrt{q^{2}-2 q \cos \phi_{1}+1}}
\end{aligned}
$$

allows equation (39) to be written as

$$
\cos \omega \cos \phi_{2}+\sin \omega \sin \phi_{2}=\frac{\sin \phi_{1}}{\sqrt{q^{2}-2 q \cos \phi_{1}+1}}
$$

or

$$
\cos \left(\phi_{2}-\omega\right)=\frac{\sin \phi_{1}}{\sqrt{q^{2}-2 q \cos \phi_{1}+1}}
$$


Since, for any angle $\theta$,

$$
\cos \theta=\cos (2 \pi-\theta)
$$

equation (43) has the two solutions

$$
\phi_{2}=\omega+\cos ^{-1} \frac{\sin \phi_{1}}{\sqrt{q^{2}-2 q \cos \phi_{1}+1}}
$$

or

$$
\phi_{2}=2 \pi+\omega-\cos ^{-1} \frac{\sin \phi_{1}}{\sqrt{q^{2}-2 q \cos \phi_{1}+1}}
$$

From equation (44), using equations (41) and (42) comes

$$
\sin \phi_{2}=\frac{\left(1-q \cos \phi_{1}\right) \sin \phi_{1}+q \sin \phi_{1} \sqrt{\left(q-\cos \phi_{1}\right)^{2}}}{q^{2}-2 q \cos \phi_{1}+1}
$$

and

$$
\cos \phi_{2}=\frac{\mathrm{q} \sin ^{2} \phi_{1}-\left(1-\mathrm{q} \cos \phi_{1}\right) \sqrt{\left(\mathrm{q}-\cos \phi_{1}\right)^{2}}}{\mathrm{q}^{2}-2 \mathrm{q} \cos \phi_{1}+1}
$$

Suppose $q>\cos \phi_{1}$, then equations (46) and (47) give

$$
\begin{aligned}
& \sin \phi_{2}=\sin \phi_{1} \\
& \cos \phi_{2}=\cos \phi_{1}
\end{aligned}
$$

But $\phi_{2}=\phi_{1}$ is specifically excluded by equation (36).

Suppose that $\mathrm{q}<\cos \phi_{1}$, equations (46) and (47) give

$$
\begin{aligned}
& \sin \phi_{2}=\frac{\left(1-q^{2}\right) \sin \phi_{1}}{q^{2}-2 q \cos \phi_{1}+1} \\
& \cos \phi_{2}=\frac{2 q-\left(1+q^{2}\right) \cos \phi_{1}}{q^{2}-2 q \cos \phi_{1}+1}
\end{aligned}
$$


The conclusion is, then, that if

$$
\mathrm{q}<\cos \phi_{1}
$$

then

$$
\phi_{2}=\omega+\cos ^{-1} \frac{\sin \phi_{1}}{\sqrt{q^{2}-2 q \cos \phi_{1}+1}}
$$

From equation (45)

$$
\begin{aligned}
& \sin \phi_{2}=\frac{\left(1-q \cos \phi_{1}\right) \sin \phi_{1}-q \sin \phi_{1} \sqrt{\left(q-\cos \phi_{1}\right)^{2}}}{q^{2}-2 q \cos \phi_{1}+1} \\
& \cos \phi_{2}=\frac{q \sin ^{2} \phi_{1}+\left(1-q \cos \phi_{1}\right) \sqrt{\left(q-\cos \phi_{1}\right)^{2}}}{q^{2}-2 q \cos \phi_{1}+1}
\end{aligned}
$$

Suppose $q>\cos \phi_{1}$, then

$$
\sin \phi_{2}=\frac{\left(1-q^{2}\right) \sin \phi_{1}}{q^{2}-2 q \cos \phi_{1}+1}
$$

and

$$
\cos \phi_{2}=\frac{2 q-\left(1+q^{2}\right) \cos \phi_{1}}{q^{2}-2 q \cos \phi_{1}+1}
$$

Now, suppose $\cos \phi_{1}>q$, then

$$
\sin \phi_{2}=\sin \phi_{1}
$$


and

$\cos \phi_{2}=\cos \phi_{1}$

And the conclusion is that if

$\cos \phi_{1}>\mathrm{q}$

then

$$
\phi_{2}=2 \pi+\omega-\cos ^{-1} \frac{\sin \phi_{1}}{\sqrt{q^{2}-2 q \cos \phi_{1}+1}}
$$

since $\phi_{1}=\phi_{2}$ is not allowable.

The term "allowable" is understood in the context that $\phi_{1}=\phi_{2}$ introduces a singularity in equation (36) so that $\mathrm{Q}_{2}$ diverges to infinity. This is, physically, allowed because it corresponds to a parabolic transfer. The case of $\phi_{2} \equiv \phi_{1}$ is rejected, however.

Equations (50) and (51) [or (54) and (55)] are always valid. Using these, the term $\sin \left(\phi_{2}-\phi_{1}\right)$ which appears in equations (34) and (36) can be written as

$$
\sin \left(\phi_{2}-\phi_{1}\right)=\frac{2 \sin \phi_{1}\left(\cos \phi_{1}-q\right)}{q^{2}-2 q \cos \phi_{1}+1}
$$

so that either of equations (34) can be combined into

$$
\mathrm{e}_{2}=\frac{ \pm \mathrm{e}_{3}\left(1-\mathrm{q}^{2}\right)}{2\left(\cos \phi_{1}-\mathrm{q}\right)-\mathrm{e}_{3}\left(\mathrm{q}^{2}-2 \mathrm{q} \cos \phi_{1}+1\right)}
$$

Using equation (37) to define $\sin \phi_{2}$ and equation (58) to define $\sin \left(\phi_{2}-\phi_{1}\right)$ equation (36), for $Q_{2}$,
becomes

$$
\mathrm{Q}_{2}=\left(\frac{\mathrm{Q}_{1}}{1+\mathrm{e}_{3} \mathrm{q}}\right)\left\{1-\mathrm{e}_{3}\left[\frac{\mathrm{q}^{2}-2 \mathrm{q} \cos \phi_{1}+1}{2\left(\cos \phi_{1}-\mathrm{q}\right)}\right]\right\}
$$


However, using the definition of $\mathrm{q}$ from equation (38) reduces this to

$$
\mathrm{Q}_{2}=\mathrm{Q}_{3}\left\{1-\frac{\mathrm{e}_{3}\left(\mathrm{q}^{2}-2 \mathrm{q} \cos \phi_{1}+1\right)}{2\left(\cos \phi_{1}-\mathrm{q}\right)}\right\}
$$

The sign ambiguity present in equation (59) can now be resolved as follows. If the initial and final orbits intersect then the perigee of the final orbit must be less than the radius of the first or the apogee of the final orbit must be greater than the radius of the first. In the first case

$$
a_{1}>a_{3}\left(1-e_{3}\right)
$$

or

$$
\frac{1}{a_{3}\left(1-e_{3}\right)}>\frac{1}{a_{1}}
$$

or

$$
\frac{1+e_{3}}{a_{3}\left(1-e_{3}^{2}\right)}>\frac{1}{a_{1}}
$$

or

$$
\mathrm{Q}_{3}\left(1+\mathrm{e}_{3}\right)>\mathrm{Q}_{1}
$$

so that

$$
\mathrm{Q}_{3} \mathrm{e}_{3}>\mathrm{Q}_{1}-\mathrm{Q}_{3}
$$

or

$$
1>q
$$

In the second case,

$$
a_{3}\left(1+e_{3}\right)>a_{1}
$$

which leads to

$$
\mathrm{q}>-1
$$


Thus, the criterion for intersecting orbits is that

$$
-1<\mathrm{q}<1
$$

The numerator of $e_{2}$ is, then, certainly positive. Consider the denominator of $e_{2}$ in equation (59). Temporarily defining

$$
f\left(\phi_{1}\right)=2\left(\cos \phi_{1}-q\right)-e_{3}\left(q^{2}-2 q \cos \phi_{1}+1\right)
$$

the extrema of $\mathrm{f}$ occur at 0 or $\pi$ so that

$$
\begin{aligned}
& f(0)=(1-q)\left[2-e_{3}(1-q)\right]>0 \\
& f(\pi)=-(1+q)\left[2+e_{3}(1+q)\right]<0
\end{aligned}
$$

and

$$
\frac{\partial f}{\partial \phi_{1}}=-2 \sin \phi_{1}\left(1+e_{3} q\right)
$$

For $|\mathrm{q}|<1, \mathrm{e}_{3}<1$,

$$
\begin{aligned}
& \frac{\partial \mathrm{f}}{\partial \phi_{1}} \leqslant 0 \quad\left(0 \leqslant \phi_{1} \leqslant \pi\right) \\
& \frac{\partial \mathrm{f}}{\partial \phi_{1}} \geqslant 0 \quad\left(\pi \leqslant \phi_{1} \leqslant 2 \pi\right)
\end{aligned}
$$

Also, f will vanish (i.e., $e_{2}$ will experience a singularity) whenever

$$
\cos \phi_{1}=\frac{2 q+e_{3}\left(1+q^{2}\right)}{2\left(1+q e_{3}\right)}
$$

i.e.,

$$
\phi_{1}=\cos ^{-1}\left[\frac{2 q+e_{3}\left(1+q^{2}\right)}{2\left(1+q e_{3}\right)}\right]
$$


or

$$
\phi_{1}=2 \pi-\cos ^{-1}\left[\frac{2 q+e_{3}\left(1+q^{2}\right)}{2\left(1+q e_{3}\right)}\right]
$$

The singularities of $\mathrm{Q}_{2}$ and $\mathrm{e}_{2}$ are not coincident. They may be ordered as follows:

$$
\mathrm{q}^{2}<1 \Longrightarrow \mathrm{q}^{2} \mathrm{e}_{3}<\mathrm{e}_{3} \Rightarrow 2 \mathrm{q}^{2} \mathrm{e}_{3}<\mathrm{e}_{3}+\mathrm{q}^{2} \mathrm{e}_{3}=\mathrm{e}_{3}\left(1+\mathrm{q}^{2}\right)
$$

Thus

$$
2 \mathrm{q}+2 \mathrm{q}^{2} \mathrm{e}_{3}<2 \mathrm{q}+\mathrm{e}_{3}\left(1+\mathrm{q}^{2}\right) \Leftrightarrow 2 \mathrm{q}\left(1+\mathrm{qe}_{3}\right)<2 \mathrm{q}+\mathrm{e}_{3}\left(1+\mathrm{q}^{2}\right)
$$

so that

$$
q<\frac{2 q+e_{3}\left(1+q^{2}\right)}{2\left(1+q e_{3}\right)}
$$

Thus, if $\phi_{1}$ begins at zero and progresses through increasing values toward $\pi$, and if $q>0$ the first singularity will be encountered at a value given by equation (65) and, subsequently, the singularity $\phi_{1}=$ $\cos ^{-1} q$ will be encountered. (In physical terms, $e_{2}$ will diverge before $Q_{2}$ diverges.) If we proceed from $2 \pi$ through negative values toward $\pi$, the same sequence occurs.

Let the upper half of the polar orbital plane $\left(0 \leqslant \phi_{1} \leqslant \pi\right)$ be divided into three regions, labeled I, II, and III, defined as follows:

I. $\phi_{1}<\cos ^{-1}\left[\frac{2 q+e_{3}\left(1+q^{2}\right)}{2\left(1+e_{3} q\right)}\right]$ and $\quad \phi_{1}<\cos ^{-1} q$

II. $\phi_{1}>\cos ^{-1}\left[\frac{2 \mathrm{q}+\mathrm{e}_{3}\left(1+\mathrm{q}^{2}\right)}{2\left(1+\mathrm{e}_{3} \mathrm{q}\right)}\right] \quad$ and $\quad \phi_{1}<\cos ^{-1} \mathrm{q}$

III. $\phi_{1}>\cos ^{-1}\left[\frac{2 q+e_{3}\left(1+q^{2}\right)}{2\left(1+e_{3} q\right)}\right]$ and $\quad \phi_{1}>\cos ^{-1} q$ 
The goal is to define physically realizable orbits over as many of these regions as the constraints allow. From $f(0)>0$ and equations (64) and (67), $e_{2}$ in region I must be positive, so choose the sign in equation (59) such that

$$
e_{2}=\frac{+e_{3}\left(1-q^{2}\right)}{2\left(\cos \phi_{1}-q\right)-e_{3}\left(q^{2}-2 q \cos \phi_{1}+1\right)}
$$

and $\rho=-\phi_{1}$. Also, for $|q|<1, Q_{2}(0)>0$. Inserting (66) into (61) gives

$$
\mathrm{Q}_{2}\left\{\cos ^{-1}\left[\frac{2 \mathrm{q}+\mathrm{e}_{3}\left(1+\mathrm{q}^{2}\right)}{2\left(1+\mathrm{q}_{3}\right)}\right]\right\}=0
$$

(i.e., parabolic transfer). Thus, region I is an area of valid orbital transfers, and similarly for the mirror image in the lower half of the plane.

In region $\mathrm{II}, \mathrm{Q}_{2}$ decreases from 0 to negative infinity, always staying negative. This region, then, does not correspond to physically realizable transfers since $\mathrm{Q}_{2}$ must be positive.

Region III again finds $Q_{2}$ to be positive since

$$
\mathrm{Q}_{2}(\pi)>0
$$

and

$$
\frac{\partial \mathrm{Q}_{2}}{\partial \mathrm{Q} \phi_{1}}<0 \quad\left(0 \leqslant \phi_{1} \leqslant \pi ; \phi_{1} \neq \cos ^{-1} \mathrm{q}\right)
$$

Since $Q_{2}$ undergoes an infinite discontinuity at $\phi_{1}=\cos ^{-1} \mathrm{q}$, these results are consistent.

The only remaining question is the behavior of $e_{2}$ in region III. From (59), certainly

$$
q^{2}-2 q \cos \phi_{1}+1>0
$$

and since $\phi_{1}>\cos ^{-1} \mathrm{q}$,

$$
\cos \phi_{1}-\mathrm{q}<0
$$


so that

$2\left(\cos \phi_{1}-q\right)-e_{3}\left(q^{2}-2 q \cos \phi_{1}+1\right)<0$

Since $1-q^{2}>0$, the conclusion is that $e_{2}$ will be positive if

$$
\mathrm{e}_{2}=\frac{-\mathrm{e}_{3}\left(1-\mathrm{q}^{2}\right)}{2\left(\cos \phi_{1}-\mathrm{q}\right)-\mathrm{e}_{3}\left(\mathrm{q}^{2}-2 \mathrm{q} \cos \phi_{1}+1\right)}
$$

from which the second of equations (34) shows that $\rho=\pi-\phi$.

The information gathered on the regions can now be summarized pictorially as follows:

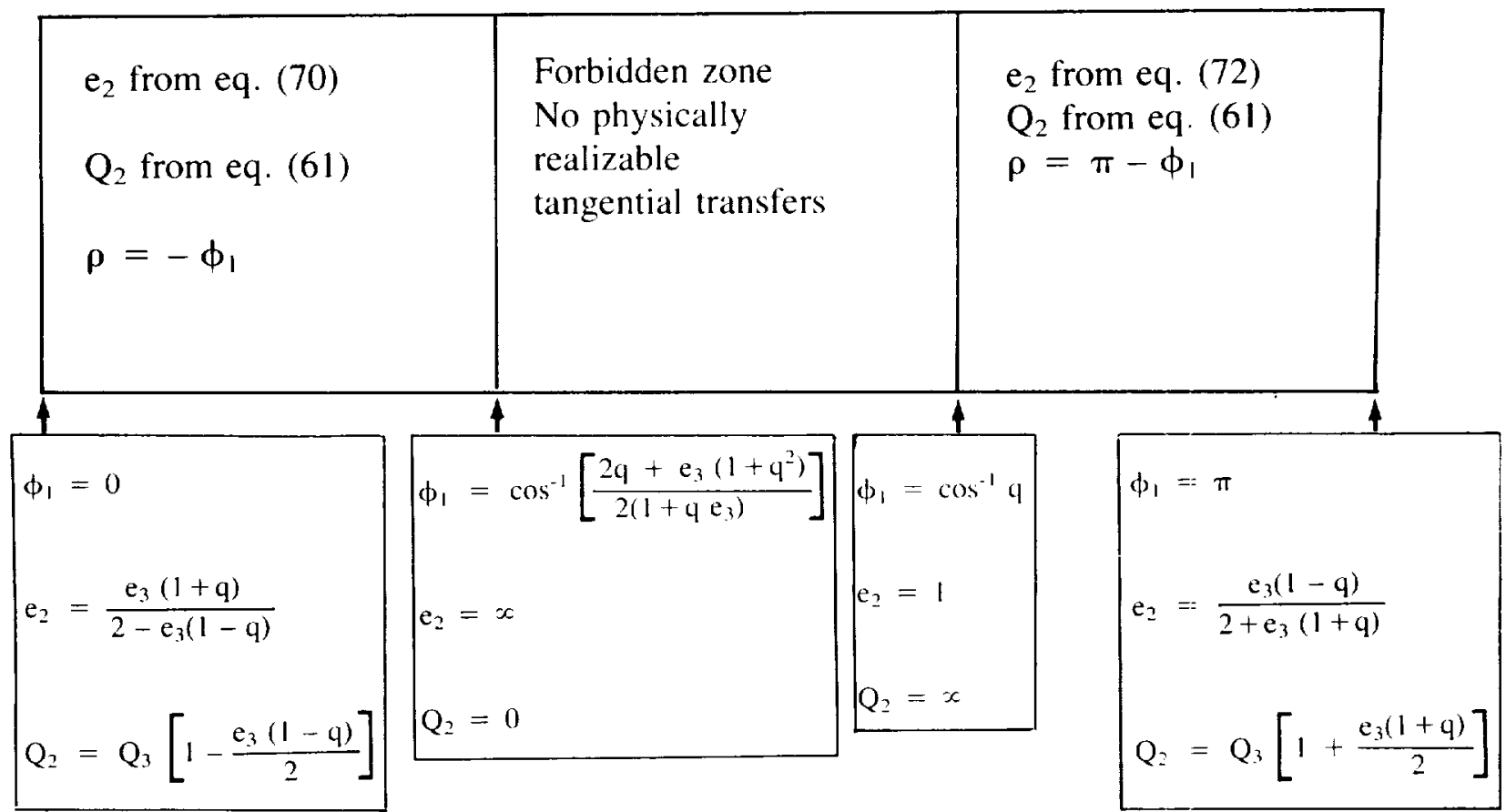

These are valid for any intersecting orbits with $\mathrm{e}_{1}=0$ and $\mathrm{e}_{3} \neq 0$, but no discussion has yet been offered for non-intersecting orbits $(|q|>1)$. That case is considerably easier to deal with than the intersecting case. The equation for $e_{2}$, equation (59), is valid but now, since $|q|>1$, there can be no singularity in $\mathrm{e}_{2}$. This is because equation (66) cannot be fulfilled $\left(\left|\cos \phi_{1}\right| \leqslant 1\right)$. 
From the bounds on $\mathrm{f}$, following equation (63),

$(1-q)\left[2-e_{3}(1-q)\right] \geqslant 2\left(\cos \phi_{1}-q\right)-e_{3}\left(q^{2}-2 q \cos \phi_{1}+1\right)>-(1+q)\left[2+e_{3}(1+q)\right]$

If $\mathrm{q}>1$, then $\mathrm{f}<0$. If $\mathrm{q}<-1$ then $\mathrm{f}>0$. This yields a simple rule since $\mathrm{l}-\mathrm{q}^{2}<0$.

If $q>1$, choose

$e_{2}=\frac{e_{3}\left(1-q^{2}\right)}{2\left(\cos \phi_{1}-q\right)-e_{3}\left(q^{2}-2 q \cos \phi_{1}+1\right)}$

If $\mathrm{q}<-1$, choose

$\mathrm{e}_{2}=\frac{-\mathrm{e}_{3}\left(1-\mathrm{q}^{2}\right)}{2\left(\cos \phi_{1}-\mathrm{q}\right)-\mathrm{e}_{3}\left(\mathrm{q}^{2}-2 \mathrm{q} \cos \phi_{1}+1\right)}$

The calculations on $Q_{2}$ are more delicate. From equation (61), if $q>1$ it is obvious that $Q_{2}>0$ for all $\phi_{2}$ and $e_{3}$. If $q<-1$ then additional considerations are needed. From equations (38) and (11) comes the relationship

$$
q=\frac{Q_{1}-Q_{3}}{Q_{3} e_{3}}=\frac{\frac{1}{a_{1}} \frac{1}{a_{3}\left(1-e_{3}^{2}\right)}}{\frac{e_{3}}{a_{3}\left(1-e_{3}{ }^{2}\right)}}=\frac{a_{3}\left(1-e_{3}{ }^{2}\right)-a_{1}}{a_{1} e_{3}}
$$

so that

$$
a_{1}=\frac{a_{3}\left(1-e_{3}^{2}\right)}{1+e_{3} q}
$$

or

$$
a_{3}=\frac{a_{1}\left(1+e_{3} q\right)}{1-e_{3}^{2}}
$$


In each case both $a_{1}$ and $a_{3}$ must be positive along with $0 \leqslant e_{3}<1$. But combinations can be chosen which violate this physical constraint. (There is no treatment here of the initial or final orbits being parabolas or hyperbolae.) The requirement, then, is that

$$
1+\mathbf{e}_{3} \mathrm{q}>0
$$

or

$-1>\mathrm{q}>-1 / \mathrm{e}_{3}, 0<\mathrm{e}_{3}<1$.

Let $\epsilon$ be a real positive number defined according to

$$
q=\epsilon^{2}-1 / e_{3}
$$

From the extremal values on $\mathrm{Q}_{2}$ comes the inequality

$$
\mathrm{Q}_{3}\left[1-\frac{\mathrm{e}_{3}(1-\mathrm{q})}{-}\right] \leqslant \mathrm{Q}_{2} \leqslant \mathrm{Q}_{3}\left[1+\frac{\mathrm{e}_{3}(1+\mathrm{q})}{2}\right]
$$

or

$$
\left(\mathrm{Q}_{3} / 2\right)\left[1-\mathrm{e}_{3}\left(1-\epsilon^{2}\right)\right] \leqslant \mathrm{Q}_{2} \leqslant\left(\mathrm{Q}_{3} / 2\right)\left[1+\mathrm{e}_{3}\left(1+\epsilon^{2}\right)\right]
$$

so that $\mathrm{Q}_{2}$ will be positive.

The conclusion that is reached is that, for the present case, there are no restrictions on tangential orbit transfers if the orbits do not intersect. 
Third Singular Case: Initial Eccentricity $\neq 0$, Final Eccentricity $=0$

This case is not identical to the previously treated case. The analogues of equations (24) through (27) are

$$
\begin{aligned}
& \mathrm{Q}_{1}\left(1+\mathrm{e}_{1} \cos \phi_{1}\right)=\mathrm{Q}_{2}\left[1+\mathrm{e}_{2} \cos \left(\phi_{1}+\rho\right)\right] \\
& \mathrm{Q}_{1} \mathrm{e}_{1} \sin \phi_{1}=\mathrm{Q}_{2} \mathrm{e}_{2} \sin \left(\phi_{1}+\rho\right) \\
& \mathrm{Q}_{2}\left[1+\mathrm{e}_{2} \cos \left(\phi_{2}+\rho\right)\right]=\mathrm{Q}_{3} \\
& \mathrm{Q}_{2} \mathrm{e}_{2} \sin \left(\phi_{2}+\rho\right)=0
\end{aligned}
$$

from which comes, immediately,

$$
\rho=-\phi_{2}
$$

or

$$
\rho=\pi-\phi_{2}
$$

In a manner exactly parallel to prior developments the following equations are obtained.

$$
\begin{aligned}
& \text { If } \rho=-\phi_{2} \\
& \mathrm{e}_{2}=\frac{\mathrm{e}_{1} \sin \phi_{1}}{\sin \left(\phi_{1}-\phi_{2}\right)-\mathrm{e}_{1} \sin \phi_{2}} \\
& \text { If } \rho=\pi-\phi_{2} \\
& \mathrm{e}_{2}=\frac{-\mathrm{e}_{1} \sin \phi_{1}}{\sin \left(\phi_{1}-\phi_{2}\right)-\mathrm{e}_{1} \sin \phi_{2}}
\end{aligned}
$$


In either case

$$
\mathrm{Q}_{2}=\mathrm{Q}_{3}\left[\frac{\sin \left(\phi_{1}-\phi_{2}\right)-\mathrm{e}_{1} \sin \phi_{2}}{\sin \left(\phi_{1}-\phi_{2}\right)+\mathrm{e}_{1}\left(\sin \phi_{1}-\sin \phi_{2}\right)}\right]
$$

The definition of $\mathrm{q}$ is changed, for this case, to read

$$
\mathrm{q}=\frac{\mathrm{Q}_{3}-\mathrm{Q}_{1}}{\mathrm{Q}_{1} \mathrm{e}_{1}}
$$

then

$$
\left(1-\mathrm{q} \cos \phi_{1}\right) \sin \phi_{2}+\left(\mathrm{q} \sin \phi_{1}\right) \cos \phi_{2}=\sin \phi_{1} .
$$

The value of $\omega$ from equation (40) has the same functional form so that equations (44) and (45) are still valid for $\phi_{2}$; similarly, for the values of $\sin \phi_{2}$ and $\cos \phi_{2}$ as given by, say, equations (50) and (51).

Since the functional form of $e_{2}$ differs, the former equation cannot be expected to hold true. Now

$$
\sin \left(\phi_{2}-\phi_{1}\right)=\frac{2 \sin \phi_{1}\left(\mathrm{q}-\cos \phi_{1}\right)}{\mathrm{q}^{2}-2 \mathrm{q} \cos \phi_{1}+1}
$$

so that from equations (83) and (84)

$$
e_{2}=\frac{ \pm e_{1}\left(q^{2}-2 q \cos \phi_{1}+1\right)}{2\left(q-\cos \phi_{1}\right)-e_{1}\left(1-q^{2}\right)}
$$

The equation for $\mathrm{Q}_{2}$, (85), becomes

$$
Q_{2}=Q_{1}\left[1-\frac{e_{1}\left(1-q^{2}\right)}{2\left(q-\cos \phi_{1}\right)}\right]
$$

Next, the sign of $\mathrm{e}_{2}$ must be determined. This will be done in the same manner as before, first for the case of intersecting orbits. 
Assume that the perigee of the initial orbit is less than the radius of the final orbit

$$
\begin{aligned}
& \mathrm{a}_{1}\left(1-\mathrm{e}_{1}\right)<\mathrm{a}_{3} \\
& \frac{1}{\mathrm{a}_{3}}<\frac{1}{\mathrm{a}_{1}\left(1-\mathrm{e}_{1}\right)} \\
& \frac{1}{\mathrm{a}_{3}}<\frac{1+\mathrm{e}_{1}}{\mathrm{a}_{1}\left(1-\mathrm{e}_{1}{ }^{2}\right)} \\
& \mathrm{Q}_{3}<\mathrm{Q}_{1}\left(1+\mathrm{e}_{1}\right) \\
& \frac{\mathrm{Q}_{3}-\mathrm{Q}_{1}}{\mathrm{Q}_{1} \mathrm{e}_{1}}<1
\end{aligned}
$$

\section{Therefore}

$$
\mathrm{q}<1
$$

Next, assume that the apogee of the initial orbit is greater than the radius of the final orbit so that

$$
\begin{aligned}
& a_{1}\left(1+e_{1}\right)>a_{3} \\
& \frac{1}{a_{3}}>\frac{1}{a_{1}\left(1+e_{1}\right)} \\
& \frac{1}{a_{3}}>\frac{1-e_{1}}{a_{1}\left(1-e_{1}^{2}\right)} \\
& Q_{3}>Q_{1}\left(1-e_{1}\right) \\
& Q_{3}-Q_{1}>-1 \\
& Q_{1} e_{1}
\end{aligned}
$$

so that the familiar bound is still

$$
-1<\mathrm{q}<1
$$


From equation (88), $\mathrm{e}_{2}$ will experience local minima at $\phi_{1}=0$ and $\phi_{1}=\pi$. Furthermore, $\mathrm{e}_{2}$ has a singularity at

$$
\phi_{1}=\cos ^{-1}\left[q-\frac{e_{1}\left(1-q^{2}\right)}{2}\right]
$$

At $\phi_{1}=0$

$$
\mathrm{e}_{2}=\frac{ \pm \mathrm{e}_{1}(\mathrm{q}-1)}{2+\mathrm{e}_{1}(1+\mathrm{q})}
$$

So to ensure a positive $e_{2}$, the negative sign is chosen. The same sign choice continues until $e_{2}$ diverges. At $\phi_{1}=\pi$

$$
\mathrm{e}_{2}=\frac{ \pm \mathrm{e}_{1}(\mathrm{q}+1)}{2+\mathrm{e}_{1}(\mathrm{q}-1)}
$$

so that in the locality of $\pi$, the positive sign is the proper choice.

$\mathrm{Q}_{2}$ [eq. (89)] will experience a singularity at

$$
\phi_{1}=\cos ^{-1} \mathrm{q}
$$

The singularities may be ordered as follows:

$$
\begin{aligned}
& 1-q^{2}>0 \\
& \frac{e_{1}}{2\left(1-q^{2}\right)} \\
& 0>\frac{-e_{1}}{2\left(1-q^{2}\right)} \\
& q>q-\frac{e_{1}}{2\left(1-q^{2}\right)}
\end{aligned}
$$


Thus, if $\phi_{1}$ begins at 0 and increases toward $\pi$, the first singularity that will be encountered occurs in $\mathrm{Q}_{2}$. At that point $\mathrm{Q}_{2}$ diverges and becomes negative. It continues as negative until the singularity in $\mathrm{e}_{2}$ is encountered. Thus, the "forbidden" region is determined by

$$
\cos ^{-1} q \leqslant \phi_{1} \leqslant \cos ^{-1} \frac{q}{q-\frac{e_{1}}{2\left(1-q^{2}\right)}}
$$

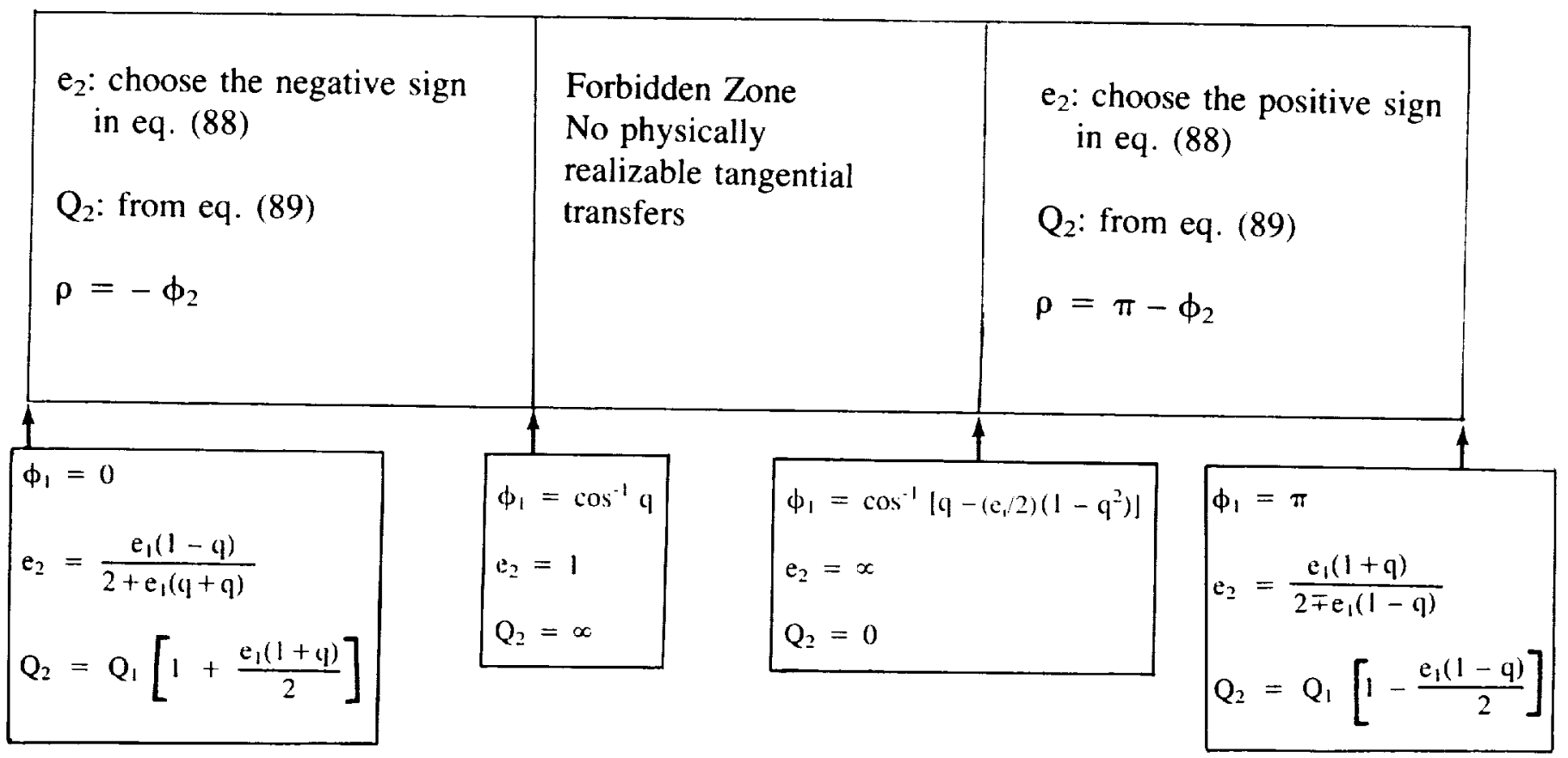

As before, there are no restrictions on the value of $\phi_{1}$ for transfer if the orbits do not intersect.

\section{The General Case: Initial Eccentricity $\neq 0$, Final Eccentricity $\neq 0$}

The prior three sections assumed that either or both of the eccentricities of the initial and final orbits vanished and this yielded much simplification. The general case will now be treated. Equations (12) through (15) constitute the natural starting point for the development.

Dividing equation (13) by equation (15) yields

$$
\frac{\sin \left(\phi_{1}+\rho\right)}{\sin \left(\phi_{2}+\rho\right)}=\frac{Q_{1} e_{1} \sin \phi_{1}}{Q_{3} e_{3} \sin \left(\phi_{2}+\vartheta\right)}
$$


Defining

$$
\gamma=\frac{Q_{1} e_{1}}{Q_{3} e_{3}}
$$

allows equation (94) to be written as

$$
\sin \left(\phi_{1}+\rho\right) \sin \left(\phi_{2}+\vartheta\right)=\gamma \sin \phi_{1} \sin \left(\phi_{2}+\rho\right)
$$

Expanding the functions which involve $\rho$, gathering, and solving for $\tan \rho$ gives

$\tan \rho=\frac{\gamma \sin \phi_{1} \sin \phi_{2}-\sin \phi_{1} \sin \left(\phi_{2}+\vartheta\right)}{\cos \phi_{1} \sin \left(\phi_{2}+\vartheta\right)-\gamma \sin \phi_{1} \cos \phi_{2}}=\sin \phi_{1}\left\{\frac{(\gamma-\cos \vartheta) \tan \phi_{2}-\sin y}{\cos \phi_{1} \cos \vartheta \tan \phi_{2}+\cos \phi_{1} \sin \vartheta-\gamma \sin \phi_{1}}\right\}$

From equations (12) and (13) comes

$$
\frac{e_{2} \sin \left(\phi_{1}+\rho\right)}{1+e_{2} \cos \left(\phi_{1}+\rho\right)}=\frac{e_{1} \sin \phi_{1}}{1+e_{1} \cos \phi_{1}}
$$

Solving for $\mathrm{e}_{2}$

$$
\mathrm{e}_{2}=\frac{\mathrm{e}_{1} \sin \phi_{1}}{\sin \left(\phi_{1}+\rho\right)+\mathrm{e}_{1} \sin \rho}
$$

Multiplying equation (12) by $\sin \left(\phi_{1}+\rho\right)$, equation (13) by $\cos \left(\phi_{1}+\rho\right)$, and adding gives

$$
\mathrm{Q}_{2}=\frac{\mathrm{Q}_{1}\left[\sin \left(\phi_{1}+\rho\right)+\mathrm{e}_{1} \sin \rho\right]}{\sin \left(\phi_{1}+\rho\right)}
$$

Equations (98) and (99) can now be used to eliminate $e_{2}$ and $Q_{2}$ from equation (14). This yields

$$
\frac{Q_{1}\left[e_{1} \sin \rho+\sin \left(\phi_{1}+\rho\right)\right]}{\sin \left(\phi_{1}+\rho\right)}\left[1+\frac{e_{1} \sin \phi_{1} \cos \left(\phi_{2}+\rho\right)}{\sin \left(\phi_{1}+\rho\right)+e_{1} \sin \rho}\right]=Q_{3}\left[1+e_{3} \cos \left(\phi_{2}+\vartheta\right)\right]
$$


Clearing shows that either

$$
\mathrm{e}_{1} \sin \rho+\sin \left(\phi_{1}+\rho\right)=0
$$

or

$$
\mathrm{Q}_{1}\left\{\sin \left(\phi_{1}+\rho\right)+\mathrm{e}_{1}\left[\sin \rho+\sin \phi_{1} \cos \left(\phi_{2}+\rho\right)\right]\right\}=\mathrm{Q}_{3}\left[1+\mathrm{e}_{3} \cos \left(\phi_{2}+\vartheta\right)\right] \sin \left(\phi_{1}+\rho\right)
$$

Comparison between equations (100) and (98) leaves only equation (102) as a valid alternative.

Now using the identity

$$
\sin \left(\phi_{1}+\rho\right)=\cos \rho\left[\sin \phi_{1}+\cos \phi_{1} \tan \rho\right]
$$

and substituting for tan $\rho$ from equation (97) into equation (103) gives

$$
\sin \left(\phi_{1}+\rho\right)=\frac{\gamma \sin \phi_{1} \sin \left(\phi_{2}-\phi_{1}\right) \cos \rho}{\cos \phi_{2}\left[\cos \phi_{1} \cos \vartheta \tan \phi_{2}+\cos \phi_{1} \sin \vartheta-\gamma \sin \phi_{1}\right]}
$$

Similarly, since

$$
\begin{aligned}
\cos \left(\phi_{2}+\rho\right) & =\cos \rho\left[\cos \phi_{2}-\sin \phi_{2} \tan \rho\right] \\
& =\frac{\left[-\gamma \sin \phi_{1}+\cos \left(\phi_{1}-\phi_{2}\right) \sin \left(\phi_{2}+\vartheta\right)\right] \cos \rho}{\cos \phi_{2}\left(\cos \phi_{1} \cos \rho \tan \phi_{2}+\cos \phi_{1} \sin \vartheta-\gamma \sin \phi_{1}\right)}
\end{aligned}
$$

A portion of the second member of equation (102) can now be constructed as

$$
\sin \rho+\sin \phi_{1} \cos \left(\phi_{2}+\rho\right)=\cos \rho\left\{\tan \rho+\frac{-\gamma \sin \phi_{1}+\cos \left(\phi_{1}-\phi_{2}\right) \sin \left(\phi_{2}+\vartheta\right)}{\cos \phi_{2}\left(\cos \phi_{1} \cos \vartheta \tan \phi_{2}+\cos \phi_{1} \sin \vartheta-\gamma \sin \phi_{1}\right)}\right\}
$$

Again substituting for $\tan \vartheta$ from equation (97) and combining fractions gives 
$\sin \rho+\sin \phi_{1} \cos \left(\phi_{2}+\rho\right)=\frac{\cos \rho \sin \phi_{1}\left\{\gamma\left(\sin \phi_{2}-\sin \phi_{1}\right)-\sin \left(\phi_{2}+\vartheta\right)\left[1-\cos \left(\phi_{1}-\phi_{2}\right)\right]\right\}}{\cos \phi_{2}\left(\cos \phi_{1} \cos \vartheta \tan \phi_{2}+\cos \phi_{1} \sin \vartheta-\gamma \sin \phi_{1}\right)}$

Next, substituting from equations (103) and (107) into equation (102) and cross-multiplying by $\cos \phi_{2}\left(\cos \phi_{1} \cos \vartheta \tan \phi_{2}+\cos \phi_{1} \sin \vartheta-\gamma \sin \phi_{1}\right)$ gives

$Q_{1} e_{1} \cos \rho \sin \phi_{1}\left\{\gamma\left(\sin \phi_{2}-\sin \phi_{1}\right)-\sin \left(\phi_{2}+\vartheta\right)\left[1-\cos \left(\phi_{1}-\phi_{2}\right)\right]+\right.$

$$
\begin{aligned}
& \frac{\gamma \sin \phi_{1} \sin \left(\phi_{2}-\phi_{1}\right) \cos \rho}{\cos \phi_{2}\left[\cos \phi_{1} \cos \vartheta \tan \phi_{2}+\cos \phi_{1} \sin \vartheta-\gamma \sin \phi_{1}\right)} \\
& =Q_{3}\left[1+e_{3} \cos \left(\phi_{2}+\vartheta\right)\right] \gamma \sin \phi_{1} \sin \left(\phi_{2}-\phi_{1}\right) \cos \rho
\end{aligned}
$$

Require that none of $\cos \rho, \sin \phi_{1}$, or $\cos \phi_{2}$ vanish. Then substitute for $\gamma$ from equation (95) on the right side of equation (108), then expand equation (108) to obtain

$e_{1} e_{3}\left\{\gamma\left(\sin \phi_{2}-\sin \phi_{1}\right)-\sin \left(\phi_{2}+\vartheta\right)\left[1-\cos \left(\phi_{1}-\phi_{2}\right)\right]\right\}+e_{3} \gamma \sin \left(\phi_{2}-\phi_{1}\right)$

$$
=e_{1} \sin \left(\phi_{2}-\phi_{1}\right)+e_{1} e_{3} \cos \left(\phi_{2}+\vartheta\right) \sin \left(\phi_{2}-\phi_{1}\right)
$$

Eliminate the remaining $\gamma$ terms from equation (109), expand the multi-angle expressions and gather on $\sin \phi_{2}, \cos \phi_{2}$ to obtain

$\left[\mathrm{Q}_{1} \mathrm{e}_{1}-\mathrm{Q}_{3} \mathrm{e}_{3} \cos \vartheta+\left(\mathrm{Q}_{1}-\mathrm{Q}_{3}\right) \cos \phi_{1}\right] \sin \phi_{2}-\left[\mathrm{Q}_{3} \mathrm{e}_{3} \sin \vartheta+\left(\mathrm{Q}_{1}-\mathrm{Q}_{3}\right) \sin \phi_{1}\right] \cos \phi_{2}$

$$
=Q_{1} e_{1} \sin \phi_{1}-Q_{3} e_{3} \sin \left(\phi_{1}+\vartheta\right)
$$

For convenience, define

$$
\begin{aligned}
& \mathrm{p}_{1}=\mathrm{Q}_{1} \mathrm{e}_{1}-\mathrm{Q}_{3} \mathrm{e}_{3} \cos \vartheta+\left(\mathrm{Q}_{1}-\mathrm{Q}_{3}\right) \cos \phi_{1}=\mathrm{Q}_{3} \mathrm{e}_{3}\left(\gamma-\cos \vartheta+\mathrm{q} \cos \phi_{1}\right) \\
& \mathrm{p}_{2}=\mathrm{Q}_{3} \mathrm{e}_{3} \sin \vartheta+\left(\mathrm{Q}_{1}-\mathrm{Q}_{3}\right) \sin \phi_{1}=\mathrm{Q}_{3} \mathrm{e}_{3}\left[\sin \vartheta+\mathrm{q} \sin \phi_{1}\right]
\end{aligned}
$$

* Letting $\sin \phi_{1}=0$ can provide a valid transfer. 


$$
\mathrm{p}_{3}=\mathrm{Q}_{1} \mathrm{e}_{1} \sin \phi_{1}-\mathrm{Q}_{3} \mathrm{e}_{3} \sin \left(\phi_{1}+\vartheta\right)
$$

where $\mathrm{q}$ is given by equation (38), not by equation (86).

Equations (111) through (113) are not independent as can be seen from

$$
\mathrm{p}_{3}=\mathrm{p}_{1} \sin \phi_{1}-\mathrm{p}_{2} \cos \phi_{1},
$$

so that equation (110) can be written as

$p_{1} \sin \phi_{2}-p_{2} \cos \phi_{2}=p_{1} \sin \phi_{1}-p_{2} \cos \phi_{1}$

Dividing equation $(115)$ by $\sqrt{p_{1}{ }^{2}+p_{2}{ }^{2}}$ and defining a new $\omega$ by

$$
\omega=\tan ^{-1}\left(p_{1} / p_{2}\right)
$$

allows equation (115) to be written as

$\cos \phi_{2} \cos \omega-\sin \phi_{2} \sin \omega=\cos \phi_{1} \cos \omega-\sin \phi_{1} \sin \omega$

or

$$
\cos \left(\phi_{2}+\omega\right)=\cos \left(\phi_{1}+\omega\right)
$$

Equation (117) has two solutions. The trivial solution is

$$
\phi_{2}=\phi_{1}
$$

but the desired solution comes via the identity

$$
\cos \left(\phi_{2}+\omega\right)=\cos \left(2 \pi-\phi_{2}-\omega\right)
$$


Then

$$
2 \pi-\phi_{1}-\omega=\phi_{1}+\omega
$$

or

$$
\phi_{2}=2(\pi-\omega)-\phi_{1}=2\left[\pi-\tan ^{-1}\left(\mathrm{p}_{1} / \mathrm{p}_{2}\right)\right]-\phi_{1}
$$

Equation (118) is the generalized form of either equations (44) and (45).

The next step is to utilize equation (118) to eliminate $\phi_{2}$ from equation (97) in order to obtain an expression for $\rho$ which depends only upon $\phi_{1} \cdot{ }^{*}$

Defining the variables

$$
\begin{aligned}
& \alpha=\tan ^{-1}\left(\frac{\sin \vartheta}{\gamma-\cos \vartheta}\right) \\
& r=\sqrt{\gamma^{2}-2 \gamma \cos \vartheta+1}
\end{aligned}
$$

naturally leads to

$$
\begin{aligned}
& \sin \vartheta=r \sin \alpha \\
& \gamma-\cos \vartheta=r \cos \alpha
\end{aligned}
$$

Then equation (97) may be expanded as

* The author wishes to thank J. R. Redus of Marshall Space Flight Center, Huntsville, Alabama, for pointing out the following transformation. 


$$
\begin{aligned}
\tan \rho= & \sin \phi_{1}\left[\frac{\gamma \sin \phi_{2}-\sin \phi_{2} \cos \vartheta-\cos \phi_{2} \sin \vartheta}{\cos \phi_{1} \sin \phi_{2} \cos \vartheta+\cos \phi_{1} \cos \phi_{2} \sin \vartheta-\gamma \sin \phi_{1} \cos \phi_{2}}\right] \\
& =\frac{r \sin \phi_{1} \sin \left(\phi_{2}-\alpha\right)}{\gamma \sin \left(\phi_{2}-\phi_{1}\right)-r \cos \left(\phi_{2}-\alpha\right)}
\end{aligned}
$$

Now, substituting for $\phi_{2}$ from equation (118) yields

$$
\begin{aligned}
\tan \rho & =\frac{r \sin \phi_{1} \sin \left[2 \pi-2 \omega-\phi_{1}-\alpha\right]}{\gamma \sin \left[2 \pi-2 \omega-2 \phi_{1}\right]-r \cos \phi_{1} \sin \left[2 \pi-2 \omega-\phi_{1}-\alpha\right]} \\
& =\frac{-r \sin \phi_{1} \sin \left(2 \omega+\phi_{1}+\alpha\right)}{-\gamma \sin \left[2\left(\omega+\phi_{1}\right)\right]+r \cos \phi_{1} \sin \left(2 \omega+\phi_{1}+\alpha\right)}
\end{aligned}
$$

For convenience, define

$$
R=\sqrt{r^{2}+2 r q \cos \left(\phi_{1}-\alpha\right)+q^{2}}
$$

Then

$$
\begin{aligned}
& \cos \omega=\frac{r \sin \alpha+q \sin \phi_{1}}{R} \\
& \sin \omega=\frac{r \cos \alpha+q \cos \phi_{1}}{R}
\end{aligned}
$$

so that

$$
\cos \left(\omega+\phi_{1}\right)=\frac{-r \sin \left(\phi_{1}-\alpha\right)}{R}
$$




$$
\begin{aligned}
& \sin \left(\omega+\phi_{1}\right)=\frac{\mathrm{r} \cos \left(\phi_{1}-\alpha\right)+\mathrm{q}}{\mathrm{R}} \\
& \cos (\omega+\alpha)=\frac{\mathrm{q} \sin \left(\phi_{1}-\alpha\right)}{\mathrm{R}} \\
& \sin (\omega+\alpha)=\frac{\mathrm{r}+\mathrm{q} \cos \left(\phi_{1}-\alpha\right)}{\mathrm{R}}
\end{aligned}
$$

Thus,

$$
\begin{aligned}
& \sin \left(2 \omega+\phi_{1}+\alpha\right)=\sin \left(\omega+\phi_{1}\right) \cos (\omega+\alpha)+\cos \left(\omega+\phi_{1}\right) \sin (\omega+\alpha)=\frac{\left(q^{2}-r^{2}\right) \sin \left(\phi_{1}-\alpha\right)}{R^{2}} \\
& \sin \left[2\left(\omega+\phi_{1}\right)\right]=2 \sin \left(\omega+\phi_{1}\right) \cos \left(\omega+\phi_{1}\right)=\frac{-2 r \sin \left(\phi_{1}-\alpha\right)\left[r \cos \left(\phi_{1}-\alpha\right)+q\right]}{R^{2}}
\end{aligned}
$$

Inserting equations (126) and (127) into equation (124) gives

$$
\tan \rho=\frac{\left[\sin \left(\phi_{1}-\alpha\right) / R^{2}\right]}{\left[\sin (\phi-\alpha) / R^{2}\right]} \quad\left[\frac{-r\left(q^{2}-r^{2}\right) \sin \phi_{1}}{2 r \gamma\left[r \cos \left(\phi_{1}-\alpha\right)+q\right]+r \cos \phi_{1}\left(q^{2}-r^{2}\right)}\right]
$$

Although cancelling $1 / \mathrm{R}^{2}$ in the numerator and denominator of equation (128) is valid, cancelling $\sin \left(\phi_{1}-\alpha\right)$ is a different matter. The reason for this is that $\sin \left(\phi_{1}-\alpha\right)$ can change sign and due to the fact that the arctangent function is multi-valued it will not yield the same value for

$$
\tan ^{-1} \frac{\mathrm{f}(\mathrm{x})}{\mathrm{g}(\mathrm{x})}
$$

as it does for

$$
\tan ^{-1} \frac{-f(x)}{-g(x)}
$$


Equation (128) can safely be written as

$$
\tan \rho=\frac{-r\left(q^{2}-r^{2}\right) \sin \phi_{1} \operatorname{sign}\left[\sin \left(\phi_{1}-\alpha\right)\right]}{\left\{2 r \gamma\left[r \cos \left(\phi_{1}-\alpha\right)+q\right]+r \cos \phi_{1}\left(q^{2}-r^{2}\right)\right\} \operatorname{sign}\left[\sin \left(\phi_{1}-\alpha\right)\right]}
$$

Eliminating $\alpha$ and $r$ from equation (129) gives the final form of equation (129) as

$$
\begin{aligned}
\tan \rho & =\frac{\left(\gamma^{2}-2 \gamma \cos \vartheta+1-\mathrm{q}^{2}\right) \sin \phi_{1} \operatorname{sign}\left[\sin \left(\phi_{1}-\alpha\right)\right]}{\left[\left(\gamma^{2}+\mathrm{q}^{2}-1\right) \cos \phi_{1}+2 \gamma\left(\mathrm{q}+\sin \vartheta \sin \phi_{1}\right)\right] \operatorname{sign}\left[\sin \left(\phi_{1}-\alpha\right)\right]} \\
& =\frac{\left(\gamma^{2}-2 \gamma \cos \vartheta+1-\mathrm{q}^{2}\right) \sin \phi_{1} \operatorname{sign}\left[\gamma \sin \phi_{1}-\cos \left(\phi_{1}-\vartheta\right)\right]}{\left[\left(\gamma^{2}+\mathrm{q}^{2}-1\right) \cos \phi_{1}+2 \gamma\left(\mathrm{q}+\sin \vartheta \sin \phi_{1}\right)\right] \operatorname{sign}\left[\gamma \sin \phi_{1}-\cos \left(\phi_{1}-\vartheta\right)\right]}
\end{aligned}
$$

The machinery is at hand to obtain an expression for $\mathrm{Q}_{2}$ in terms of fundamental variables. From equation (99) comes

$$
\begin{aligned}
\mathrm{Q}_{2} & =\mathrm{Q}_{1}\left\{1+\frac{\mathrm{e}_{1} \sin \rho}{\sin \phi_{1} \cos \rho+\cos \phi_{1} \sin \rho}\right\} \\
& =\mathrm{Q}_{1}\left\{1+\frac{\mathrm{e}_{1} \tan \rho}{\sin \phi_{1}+\cos \phi_{1} \tan \rho}\right\}
\end{aligned}
$$
expression

Using equation (130) (and ignoring the sign terms which make no difference here) yields the
sion

$$
\sin \phi_{1}+\cos \phi_{1} \tan \rho=2 \gamma \sin \phi_{1}\left[\frac{\gamma \cos \phi_{1}+q-\cos \left(\phi_{1}+\vartheta\right)}{\left(\gamma^{2}+q^{2}-1\right) \cos \phi_{1}+2 \gamma\left(q+\sin \vartheta \sin \phi_{1}\right)}\right]
$$

Then

$$
\mathrm{Q}_{2}=\mathrm{Q}_{1}\left\{1+\frac{\mathrm{e}_{1}\left(\gamma^{2}-2 \gamma \cos \vartheta+\mathrm{I}-\mathrm{q}^{2}\right)}{2 \gamma\left[\gamma \cos \phi_{1}+\mathrm{q}-\cos \left(\phi_{1}+\vartheta\right)\right]}\right\}
$$

is a fundamental expression for $Q_{2}$. 
The final equation which is needed is the expression for $e_{2}$ which involves only fundamental variables, i.e., a non-sequential calculation. I have been unable to derive an expression which is aesthetically satisfactory, but such an expression is not required in what follows.

The "forbidden regions" which constitute the focus of this paper exist in the general case and can be analytically defined by enforcing the following conic section restrictions:

$$
\begin{array}{ll} 
& {\left[\left(0<e_{2}<1\right) \text { and }\left(a_{2}>0\right)\right]} \\
\text { or } & {\left[\left(e_{2}>1\right) \text { and }\left(a_{2}<0\right)\right]} \\
\text { or } & {\left[\left(e_{2}=0\right) \text { and }\left(a_{2}>0\right)\right]} \\
\text { or } & {\left[\left(e_{2}=1\right) \text { and }\left(a_{2}=x\right)\right]}
\end{array}
$$

It will be shown that $e_{2}$ and $a_{2}$ can take on any value on the extended reals and the regions which violate equation (134) give rise to forbidden regions.

As in the special cases, the apparent dependency of $\operatorname{sign}\left(e_{2}\right)$ on $\operatorname{sign}\left[\sin \left(\phi_{1}\right)\right]$ is illusory [equation (98) 1 . Consider these points at which $e_{2}$ will experience a singularity, i.e., the solutions of

$$
\sin \left(\phi_{1}+\rho\right)+e_{1} \sin \rho=0
$$

which expands to give

$$
\begin{aligned}
& \sin \left(\phi_{1}+\rho\right)+e_{1} \sin \rho=\left[\left(\gamma^{2}+q^{2}-1\right) \cos \phi_{1}+2 \gamma\left(q+\sin \vartheta \sin \phi_{1}\right)\right] \sin \phi_{1} \\
& \quad+\left[\left(\gamma^{2}-2 \gamma \cos \vartheta+1-q^{2}\right)\right] \cos \phi_{1} \sin \phi_{1}+e_{1}\left(\gamma^{2}-2 \gamma \cos \vartheta+1-q^{2}\right) \sin \phi_{1}=0
\end{aligned}
$$

Since $\sin \phi_{1}$ is assumed not to vanish, gather on $\sin \phi_{1}, \cos \phi_{1}$, to yield

$$
(\gamma-\cos \vartheta) \cos \phi_{1}+\sin \vartheta \sin \phi_{1}=\left\{\frac{e_{1}\left[2 \gamma \cos \vartheta+q^{2}-\gamma^{2}-1\right]-2 \gamma q}{2 \gamma}\right\}
$$

Utilizing the definitions of $\alpha$ from equation (119) and defining

$$
\zeta=\cos ^{-1}\left\{\frac{e_{1}\left[2 \gamma \cos \vartheta+q^{2}-\gamma^{2}-1\right]-2 \gamma q}{2 \gamma \sqrt{\gamma^{2}-2 \gamma \cos \vartheta+1}}\right\}
$$


then equation (137) has two solutions

$$
\begin{aligned}
& \phi_{1}=\alpha+\zeta \\
& \phi_{1}=2 \pi+\alpha-\zeta
\end{aligned}
$$

as singular points of $e_{2}$.

Another complicating factor to the orbit transfer arises when the numerator and denominator of equation (130) vanish simultaneously. These points can be isolated as follows.

Assume that the numerator of equation (97) vanishes [this is more convenient than working with equation (130)]. Then

$$
(\gamma-\cos \vartheta) \tan \phi_{2}-\sin \vartheta=0
$$

so that

$$
\tan \phi_{2}=\tan \alpha
$$

If the denominator of equation (97) also vanishes

$$
\cos \phi_{1} \cos \vartheta \tan \phi_{2}+\cos \phi_{1} \sin \vartheta-\gamma \sin \phi_{1}=0
$$

Eliminating $\phi_{2}$ between equations (141) and (142) gives

$$
\tan \phi_{1}=\frac{\sin \vartheta}{\gamma-\cos \vartheta}=\tan \alpha
$$

Since

$$
\tan \phi_{1}=\tan \left(\pi+\phi_{1}\right)
$$


the two solutions of equation (143) are

$$
\begin{aligned}
& \phi_{1}=\phi_{2}=\alpha \\
& \phi_{1}=\phi_{2}=\pi+\alpha
\end{aligned}
$$

which are also the points at which $\sin \left(\phi_{1}-\alpha\right)=0$. Thus, when the numerator and denominator of equation (97) $[$ or $(130)]$ vanish, $\operatorname{sign}\left[\sin \left(\phi_{1}-\alpha\right)\right]$ loses meaning since zero is unsigned.

Both the numerator and denominator are continuous through the points given by equation (144) and the angle $\rho$ will experience a jump discontinuity of $\pi$. The behavior of $e_{2}$ at this point is demonstrated as follows. Let $\phi^{-}$and $\phi^{+}$represent $\phi$ to the left and right of the solution given by equation (144). Then

$$
\begin{gathered}
e_{2}\left(\phi^{-}\right)=\frac{e_{1} \sin \phi^{-}}{\sin \left(\phi^{-}+\rho\right)+e_{1} \sin \rho} \\
e_{2}\left(\phi^{+}\right)=\frac{e_{1} \sin \phi^{+}}{\sin \left(\phi^{+}+\rho \pm \pi\right)+e_{1} \sin (\rho \pm \pi)} \\
\sin (\phi+\rho \pm \pi)+e_{1} \sin (\rho \pm \pi)=\cos ( \pm \pi)\left[\sin (\phi+\rho)+e_{1} \sin \rho\right] \\
=-\left[\sin (\phi+\rho)+e_{1} \sin \rho\right] \\
\left|e_{2}\left(\phi^{-}\right)\right|=-\left|e_{2}\left(\phi^{+}\right)\right|
\end{gathered}
$$

This far, $\mathrm{e}_{2}$ has exhibited two unbounded discontinuities [see equations (139)] and two bounded discontinuities [see equations (144)]. Additional information on $e_{2}$ comes from the observation that if

$$
\begin{aligned}
& \phi_{1}+\rho=\pi \\
& \mathrm{e}_{2}=1
\end{aligned}
$$

(i.e., parabolic transfer) and if

$$
\phi_{1}+\rho=0
$$


then

$e_{2}=-1$

To isolate the value of $\phi_{1}$ which corresponds to equation (145), observe that, necessarily

$\tan \rho=-\tan \phi_{1}$

Inserting this result into equation (130) gives

$\left(\gamma^{2}+q^{2}-1\right) \cos \phi_{1}+2 \gamma\left(q+\sin \vartheta \sin \phi_{1}\right)=-\left(\gamma^{2}-2 \gamma \cos \vartheta+1-q^{2}\right) \cos \phi_{1}$

Gathering, utilizing $\alpha$ once more and defining

$$
\xi=\cos ^{-1} \frac{-q}{\sqrt{\gamma^{2}-2 \gamma \cos \vartheta+1}}
$$

then equation (149) has the two solutions

$$
\begin{aligned}
& \phi_{1}=\alpha+\xi \\
& \phi_{1}=2 \pi+\alpha-\xi
\end{aligned}
$$

The physical identification of the angles given by equation (151) is worthwhile. Suppose that equation (118) is valid and, simultaneously, the trivial solution is valid so that

$$
\phi_{1}=\phi_{2}
$$

This corresponds, of course, ${ }^{*}$ to orbital intersection. Then equation $(118)$ requires that

$$
\tan \phi_{1}=-p_{1} / p_{2}
$$

* The same results can be obtained by equating the left member of equation (12) to the right member of equation (14). 
Inserting $p_{1}$ and $p_{2}$ from equations (111) and (112) yields

$$
(\gamma-\cos \vartheta) \cos \phi_{1}+\sin \vartheta \sin \phi_{1}=-q
$$

which is the reduced form of equation (149). Thus, at an orbital intersection, $e_{2}= \pm 1$.

The orbital transfer plane is cleaved into two regions of positive $e_{2}$ and two regions of negative $e_{2}$ so it is reasonable to suspect that there may be four places where $\left|e_{2}\right|=1$. Thus far, only two such points have been isolated. Two others can be obtained as follows. Setting $\left|e_{2}\right|=1$ in equations (12) and (13) yields the pair

$$
\begin{aligned}
& Q_{1}\left(1+e_{1} \cos \phi_{1}\right)=Q_{2}\left[1 \pm \cos \left(\phi_{1}+\rho\right)\right] \\
& Q_{1} e_{1} \sin \phi_{1}= \pm Q_{2} \sin \left(\phi_{1}+\rho\right)
\end{aligned}
$$

Isolating $\sin \left(\phi_{1}+\rho\right)$ and $\cos \left(\phi_{1}+\rho\right)$, then squaring and adding the resulting equations gives

$$
\mathrm{Q}_{1}\left(1+2 \mathrm{e}_{1} \cos \phi_{1}+\mathrm{e}_{1}{ }^{2}\right)=2 \mathrm{Q}_{2}\left(1+\mathrm{e}_{1} \cos \phi_{1}\right)
$$

Inserting $\mathrm{Q}_{2}$ from equation (133) and cancelling like terms gives

$\left[\mathrm{e}_{1}^{2}\left(\mathrm{q}^{2}+\gamma \cos \vartheta-1\right)-\gamma(\gamma-\cos \vartheta)\right] \cos \phi_{1}+\gamma\left(\mathrm{e}_{1}^{2}-1\right) \sin \vartheta \sin \phi_{1}$

$$
\left.=\mathrm{e}_{1}\left(\gamma^{2}-2 \gamma \cos \vartheta+1-\mathrm{q}^{2}\right)-\mathrm{q} \gamma\left(\mathrm{e}_{1}^{2}-1\right)\right]
$$

Defining

$$
\sigma=\tan ^{-1} \frac{\gamma\left(e_{1}^{2}-1\right) \sin \vartheta}{e_{1}^{2}\left(q^{2}+\gamma \cos \vartheta-1\right)-\gamma(\gamma-\cos \vartheta)}
$$

and

$$
\tau=\cos ^{-1} \frac{e_{1}\left(\gamma^{2}-2 \gamma \cos \vartheta+1-q^{2}\right)-q \gamma\left(e_{1}{ }^{2}-1\right)}{\sqrt{\left[e_{1}{ }^{2}\left(q^{2}+\gamma \cos \vartheta-1\right)-\gamma(\gamma-\cos \vartheta)\right]^{2}+\left[\gamma\left(e_{1}{ }^{2}-1\right) \sin \vartheta\right]^{2}}}
$$


then the other two points of unity for $e_{2}$ are given by the solution of equation (155) as

$$
\begin{aligned}
& \phi_{1}=\sigma+\tau \\
& \phi_{1}=2 \pi+\sigma-\tau .
\end{aligned}
$$

Within this section the behavior of $e_{2}\left(\phi_{1}\right)$ has been a focal point while $Q_{2}\left(\phi_{1}\right)$ has received less attention. The next step is to examine $Q_{2}\left(\phi_{1}\right)$ at each point which has proven to be of interest in $e_{2}\left(\phi_{1}\right)$.

The first such point pair is given by equations (139). The easiest way to make the substitution is by
equation (137) as writing equation (137) as

$$
\frac{\mathrm{e}_{1}\left(\gamma^{2}-2 \gamma \cos \vartheta+1-q^{2}\right)}{2 \gamma}=-q-(\gamma-\cos \vartheta) \cos \phi_{1}-\sin \vartheta \sin \phi_{1}
$$

and substituting the left side of equation (159) into equation (133) which immediately yields

$$
\mathrm{Q}_{2}=0
$$

at this point. This value then shows that a hyperbola of infinite eccentricity has resulted, i.e., a straight line. The next important point pair comes from equations (144), the point of finite jump discontinuity in
$\mathrm{e}_{2}$. From equation (144) comes

$$
\begin{aligned}
& \sin \phi_{1}=\frac{ \pm \sin \vartheta}{\sqrt{\gamma^{2}-2 \gamma \cos \vartheta+1}} \\
& \cos \phi_{1}=\frac{ \pm(\gamma-\cos \vartheta)}{\sqrt{\gamma^{2}-2 \gamma \cos \vartheta+1}}
\end{aligned}
$$

so that equation (133) becomes

$$
\mathrm{Q}_{2}=\mathrm{Q}_{1}\left\{1 \pm\left(\mathrm{e}_{1} / 2 \gamma\right)\left[\sqrt{\gamma^{2}-2 \gamma \cos \vartheta+1} \mp \mathrm{q}\right]\right\}
$$

where the positive sign corresponds to the $\phi_{1}=\alpha$ and the negative sign corresponds to $\phi_{1}=\pi+\alpha$. 
The first case where $\left|e_{2}\right|=1$ comes from equations (145) and (147). In either case, equation (99) shows a divergence of $\mathrm{Q}_{2}$.

The remaining case occurs when $\phi_{1}$ is given by either of equations (158). The actual value of $\mathrm{Q}_{2}$ at this point is not difficult to determine, numerically. But a single equation which gives that value is quite messy. Since the coefficient of $\mathrm{Q}_{2}$ in equation (154) cannot vanish, this point is a regular point.

The following table, Table I, summarizes the information presented here on the behavior of $e_{2}\left(\phi_{1}\right)$ and $Q_{2}\left(\phi_{1}\right)$.

TABLE I

\begin{tabular}{|c|l|l|l|}
\hline $\begin{array}{c}\text { Governing } \\
\text { Equations }\end{array}$ & Value of $\phi_{1}$ & \multicolumn{1}{|c|}{ Value of $\mathrm{e}_{2}\left(\phi_{1}\right)$} & \multicolumn{1}{c|}{ Value of $\mathrm{Q}_{2}\left(\phi_{1}\right)$} \\
\hline 139 & $\alpha+\zeta$ & Diverges & 0 \\
& $2 \pi+\alpha-\zeta$ & Diverges & 0 \\
\hline 144 & $\alpha$ & $\frac{ \pm \mathrm{e}_{1} \sin \phi_{1}}{\sin \left(\phi_{1}+\rho\right)+\mathrm{e}_{1} \sin \rho}$ & $\mathrm{Q}_{1}\left\{1+\left(\mathrm{e}_{1} / 2 \gamma\right)\left[\sqrt{\gamma^{2}-2 \gamma \cos \vartheta+1}-q \mid\right\}\right.$ \\
& $\pi+\alpha$ & $($ Jump Discontinuity) & $\mathrm{Q}_{1}\left\{1-\left(\mathrm{e}_{1} / 2 \gamma\right)\left(\sqrt{\gamma^{2}-2 \gamma \cos \vartheta+1}+q\right)\right\}$ \\
\hline 151 & $\alpha+\xi$ & \pm 1 & Diverges \\
& $2 \pi+\alpha-\xi$ & \pm 1 & Diverges \\
\hline 158 & $\sigma+\tau$ & \pm 1 & Regular Point \\
\hline
\end{tabular}


It is very convenient to order the angles which are presented in Table I. That is, for a given input of the primitive variables, predict the order in which the important points of $e_{2}$ will occur. It must be realized that certain conventions are inherent in the derivation of the prior equations -i.e., all angles must be chosen in the range $[0,2 \pi]$, the principle value of $\cos ^{-1}()$ will lie in the range $[0, \pi]$, etc. These conventions follow from standard computer implementations of the derived equations and we make use of them in the following discussion.

Since all angles are calculated in a modulo $2 \pi$ system, any angle in column 2 of Table I can exceed any other angle. Arbitrarily, but without loss of generality, choose $[0<\alpha<\pi]$. Then

$$
\alpha+\pi>\alpha>0
$$

Because of the principle value condition, equation (138) will yield

$$
\pi>\zeta \geqslant 0
$$

so that

$$
2 \pi>2 \pi-\zeta>\pi
$$

and

$$
2 \pi+\alpha>2 \pi+\alpha-\zeta>\pi+\alpha,
$$

since $2 \pi+\alpha=\alpha$ and $\pi+\alpha=\alpha-\pi$

$$
\alpha>2 \pi+\alpha-\zeta>\pi+\alpha=\alpha-\pi .
$$

The next ordering occurs between the angles $\xi$ and $\zeta$. For $\xi$ to be real, equation (150) shows that

$$
-q \leqslant \sqrt{\gamma^{2}-2 \gamma \cos \vartheta+1} .
$$

For convenience, abbreviate, as before,

$$
r=\sqrt{\gamma^{2}-2 \gamma \cos \vartheta+1} .
$$


Then

$$
\mathrm{q}^{2} \leqslant \mathrm{r}^{2},
$$

so that

$$
e_{1}\left(q^{2}-r^{2}\right) \leqslant 0
$$

and

$$
e_{1}\left(q^{2}-r^{2}\right)-2 \gamma q \leqslant-2 \gamma q,
$$

or

$$
\frac{e_{1}\left(q^{2}-r^{2}\right)-2 \gamma q}{2 \gamma r} \leqslant-\frac{q}{r}
$$

so that

$$
\cos ^{-1}(-q / r)>\cos ^{-1} \frac{e_{1}\left(q^{2}-r^{2}\right)-2 \gamma q}{2 \gamma r}
$$

From equations (150) and (138) comes

$$
\begin{aligned}
& \xi \leqslant \zeta \\
& \xi+\alpha \leqslant \zeta+\alpha \\
& 2 \pi+\alpha-\zeta \leqslant 2 \pi+\alpha-\xi .
\end{aligned}
$$


The angle ordering, thus far, appears as

$$
\alpha<\alpha+\xi<\alpha+\zeta<\alpha+\pi<2 \pi+\alpha-\zeta<2 \pi+\alpha-\xi
$$

By construction, the remaining two important angles $\sigma+\tau$ and $2 \pi+\sigma-\tau$ can only occur in one of two locations. $\sigma+\tau$ or $2 \pi+\sigma-\tau$ must occur between $2+\pi$ and $2 \pi+\alpha-\zeta$ or between $\alpha+\zeta$ and $\alpha+\pi$. Whichever angle is established for the first slot determines that the second angle will occupy the second slot. Thus, we need only establish a single inequality for a chosen configuration to determine a complete ordering. Choose $\alpha=\pi$ so that $\alpha-\pi=0$. From equation (119), $\vartheta=0$. If $116=0$, then (156) shows that $\sigma=0$. Now because of principle value considerations, $\pi \geqslant \tau$ so that

$$
2 \pi \geqslant 2 \tau
$$

or

$$
2 \pi-\tau \geqslant \tau
$$

and

$$
2 \pi+\sigma-\tau \geqslant \sigma+\tau
$$

Thus, the cyclic ordering can be written as follows:

$$
(\alpha+\pi<\sigma+\tau<2 \pi+\alpha-\zeta<2 \pi+\alpha-\xi<\alpha<\alpha+\xi<\alpha+\zeta<2 \pi+\sigma-\tau)_{\bmod } 2 \pi
$$

The computation algorithm is quite simple. Choose the minimum value of any of the important points and arrange the rest in accord with the above ordering.

The above theory has shown that certain regions of intersecting orbits preclude tangential transfers. It is intuitively obvious, however, that these forbidden regions cannot occur in the case of non-intersecting orbits. It is important to demonstrate that if the orbits do not intersect then there are no discontinuities in $\mathrm{e}_{2}\left(\phi_{1}\right)$.

Consider the following, since $\mathrm{Q}_{1}$ and $\mathrm{Q}_{3}$ are both positive

$$
\mathrm{Q}_{1}>\mathrm{Q}_{1}-\mathrm{Q}_{3}
$$


so that

$$
\frac{Q_{1} e_{1}}{Q_{3} e_{3}}>\frac{\left(Q_{1}-Q_{3}\right) e_{1}}{Q_{3} e_{3}}
$$

i.e.,

$$
\begin{aligned}
& \gamma>e_{1} q \\
& 2 \gamma>2 e_{1} q=e_{1} q+e_{1} q .
\end{aligned}
$$

Now suppose that an intersection does not occur. Then equation (150) shows that $\xi$ will not be real, i.e.,

$$
(-\mathrm{q}>\mathrm{r}) \text { or }(-\mathrm{q}<-\mathrm{r})
$$

Thus, if $-q>r$,

$$
2 \gamma>e_{1} q-e_{1} r=e_{1}(q-r)
$$

and

$$
0>(r+q)
$$

so that

$$
e_{1}(q-r)(q+r)>2 \gamma(r+q)
$$

or

$$
\frac{e_{1}\left(q^{2}-r^{2}\right)-2 \gamma q}{2 \gamma r}>1
$$




$$
\begin{aligned}
& \text { Conversely, if }-\mathrm{q}<-r \text { then } \mathrm{q}-\mathrm{r}>\text { so that } \\
& \frac{\mathrm{e}_{1}\left(\mathrm{q}^{2}-\mathrm{r}^{2}\right)-2 \gamma \mathrm{q}}{2 \gamma \mathrm{r}}<-1
\end{aligned}
$$

From (138) it is now apparent that $e_{2}$ has no infinite discontinuities in the real plane. It is also easy to demonstrate that the finite discontinuities are precluded for non-intersecting orbits. In equation (130), the numerator cannot generally vanish via $r^{2}=q^{2}$ so, if the only way it could vanish is via $\sin \phi_{1}=0$. If this is
the case then the denominator will become

$$
f= \pm\left(\gamma^{2}+q^{2}-1\right)+2 \gamma q
$$

which can be written as

$$
f= \pm(\gamma \pm q)^{2} \mp 1
$$

Suppose that $f$ does vanish. Substituting for $\gamma$ and $q$ in terms of primitive values shows that one of the four conditions

$$
\begin{aligned}
& a_{1}\left(1+e_{1}\right)=a_{3}\left(1+e_{3}\right) \\
& a_{1}\left(1-e_{1}\right)=a_{3}\left(1+e_{3}\right) \\
& a_{1}\left(1+e_{1}\right)=a_{3}\left(1-e_{3}\right) \\
& a_{1}\left(1-e_{1}\right)=a_{3}\left(1-e_{3}\right)
\end{aligned}
$$

which correspond to osculating contacts between the orbits, i.e., single impulse transfers.

With respect to $\mathrm{Q}_{2}\left(\phi_{1}\right)$, this function will diverge if the denominator of equation (133) vanishes. This leads immediately to the requirement that equation (150) can be satisfied which is contrary to hypothesis. It remains only to be shown that $\mathrm{Q}_{2}\left(\phi_{1}\right)$ is everywhere positive if there is no intersection.

Again using the generic function $\mathrm{f}$, let

$$
f=\gamma \cos \phi_{1}+q-\cos \left(\phi_{1}+\vartheta\right)
$$

so that extrema of $f$ occur when $\phi_{1}=\alpha$, i.e., 


$$
\begin{aligned}
& \cos \phi_{1}= \pm \frac{(\gamma-\cos \vartheta)}{r} \\
& \sin \phi= \pm \frac{\sin \vartheta}{r}
\end{aligned}
$$

Then the extrema of $f$ are given by

$$
\mathrm{f}_{\mathrm{ex}}= \pm \mathrm{r}+\mathrm{q}
$$

so that, from (133), the extrema of $\mathrm{Q}_{2}\left(\phi_{1}\right)$ are

$$
Q_{2, \mathrm{ex}}=Q_{1}\left[1+\frac{\mathrm{e}_{1}(\mathrm{r}-\mathrm{q})(\mathrm{r}+\mathrm{q})}{2 \gamma( \pm \mathrm{r}+\mathrm{q})}\right]
$$

Suppose, first, that

$$
Q_{2 . e x}=Q_{1}\left[1+\frac{e_{1}(x-q)}{2 \gamma}\right]
$$

and if $q=r+\varepsilon^{2}$, then

$$
\mathrm{Q}_{2, \mathrm{cx}}=\mathrm{Q}_{1}\left[1-\frac{\mathrm{e}_{1} \varepsilon^{2}}{2 \gamma}\right] \rightarrow \mathrm{Q}_{1}
$$

as $\varepsilon$ becomes small, while if $-\mathrm{q}=\mathrm{r}+\varepsilon^{2}$ then

$$
\mathrm{Q}_{2, \mathrm{ex}}=\mathrm{Q}_{1}\left[1+\frac{\mathrm{e}_{1}\left(-2 \mathrm{q}-\varepsilon^{2}\right)}{2 \gamma}\right] \rightarrow \mathrm{Q}_{1}\left[1-\frac{\mathrm{e}_{1} \mathrm{q}}{\gamma}\right]=\mathrm{Q}_{1}\left[1-\frac{\mathrm{e}_{1}\left(\mathrm{Q}_{1}-\mathrm{Q}_{3}\right) /\left(\mathrm{Q}_{3} \mathrm{e}_{3}\right)}{\left(\mathrm{e}_{1} \mathrm{Q}_{1}\right) /\left(\mathrm{Q}_{3} \mathrm{e}_{3}\right)}\right]=\mathrm{Q}_{3}
$$

as $\varepsilon$ becomes small. 
Next, let

$$
Q_{2 . \mathrm{cx}}=\mathrm{Q}_{1}\left[1-\frac{\mathrm{e}_{1}(\mathrm{r}+\mathrm{q})}{2 \gamma}\right]
$$

and $\mathrm{q}=\mathrm{r}+\varepsilon^{2}$ then

$$
\mathrm{Q}_{2 . \mathrm{x} \times}=\mathrm{Q}_{1}\left[1-\frac{\mathrm{e}_{1}\left(2 \mathrm{q}-\varepsilon^{2}\right)}{2 \gamma}\right] \rightarrow \mathrm{Q}_{1}\left[1-\frac{\mathrm{e}_{1} \mathrm{q}}{\gamma}\right]=\mathrm{Q}_{1}\left[1-\frac{\mathrm{e}_{1}\left(\mathrm{Q}_{1}-\mathrm{Q}_{3}\right) /\left(\mathrm{Q}_{3} \mathrm{e}_{3}\right)}{\left(\mathrm{Q}_{1} \mathrm{e}_{1}\right) /\left(\mathrm{Q}_{3} \mathrm{e}_{3}\right)}\right]=\mathrm{Q}_{3}
$$

as $\varepsilon$ becomes small, while if $-\mathrm{q}=\mathrm{r}+\varepsilon^{2}$ then

$$
\mathrm{Q}_{2, \mathrm{x}}=\mathrm{Q}_{1}\left[1+\frac{\mathrm{e}_{1} \varepsilon^{2}}{2 \gamma}\right] \rightarrow \mathrm{Q}_{1}
$$

as $\varepsilon$ becomes small.

The conclusion is, then, that if the orbits do not intersect, $\mathrm{Q}_{2}$ is bounded between the (positive) values of $Q_{1}$ and $Q_{3}$. Since non-intersecting orbits have no singularities in the $e_{2}\left(\phi_{1}\right)$ function and positive $\mathrm{Q}_{2}\left(\phi_{1}\right)$ values, we conclude that tangential transfer is possible between any two non-intersecting orbits. 


\section{REFERENCES}

1. Wen, W. Li-Shu: "A Study of Cotangential, Elliptical Transfer Orbits in Space Flight." J. Aerospace Sci., Vol. 28, 1961, pp. 411-417.

2. Bender, D.: "Optimum Coplanar Two Impulse Transfers Between Elliptic Orbits." North American Aviation, Inc., Space and Information Systems Division, NAA SID, 1962, pp. 46-62.

3. Marec, J.-P.: “Optimal Space Trajectories.” Elsevier Scientific, Amsterdam. 1979.

4. Busemann, A., Vinh, N.X., and Culp, R.D.: "Geometric Constraints of the Disorbit Problem (Summary)." Space Flight Mechanics Specialist Symposium, AAS Science and Technology Series (Edited by M.L. Anthony), Vol. 11, AAS Publications Office, Tarzana, California.

5. Burns, R.E.: "Forbidden Tangential Orbit Transfers Between Intersecting Keplerian Orbits." Acta Astronautica, Vol. 19, No. 8, 1989, pp. 649-656. 


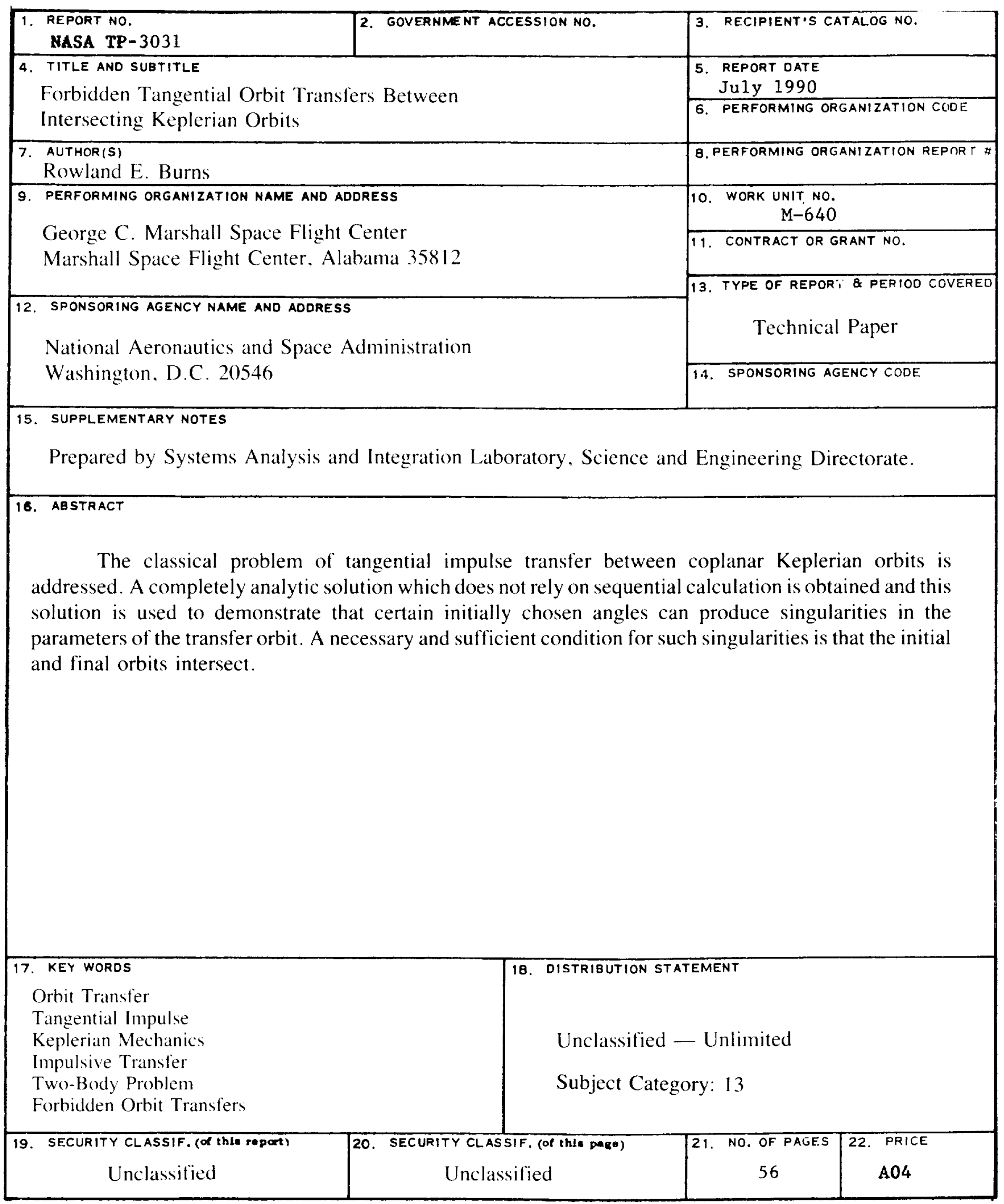


